\title{
Finnish Chironomidae
}

\author{
Lauri Paasivirta \\ Ruuhikoskenkatu 17, B 5, 24240 Salo, Finland \\ E-mail: lauri.paasivirta@suomi24.fi
}

The current list of Chironomidae from Finland comprises 781 species altogether (Table 1). This includes 41 undescribed species, 12 'problematic' species (cf. or ?), 1 exuvial and 4 larval types. Compared to the previous version from March $20^{\text {th }} 2009,1015$ additions and 23 removals in the provincial records have been made.

Regional and national check-lists are often based on records from a large number of scientists with variable knowledge of both species distributions and taxonomy. For the list of species from Finland, over $90 \%$ of the records are now my own identifications. These are mainly of own sweep-net samples (74\%) from the period 1967 - 2012 and the massive whole-season Malaise-trap sampling by esp. Jukka Salmela during the last ten years ( $71 \%$ of all Malaise-trap samples). Some additional records come from collections of exuviae (mainly by Janne Raunio) and larvae (mainly by me). An overview of the sampling efforts divided by bioprovinces (Figure 1) and method is given in table 2.

The material is deposited mainly in my own collection consisting of thousands of slides and alcohol vials. The hypopygia of the undescribed spp. have been photographied and drawn. DNA barcoding using partial cytochrome c oxidase subunit 1 (COI) sequences has been done on 226 specimens of 77 species from southwest Finland, including two types in Procladius choreus-agg. and three types in Chironomus pseudothummi- agg. Papers giving summaries of the local fauna in the main habitat types are now in preparation.

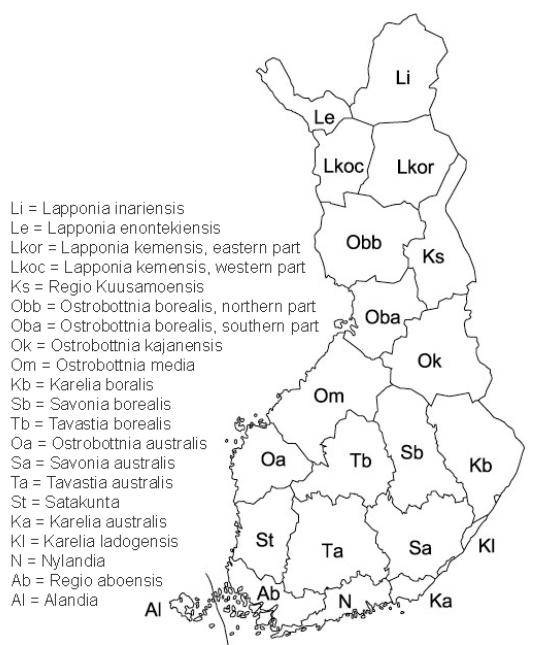

Figure 1. The bioprovinces in Finland.
Table 2. Number of species by sampling efforts in bioprovinces. 14 light-trap nights are included in the sweep-net samples. Most Malaise-traps were operated through the whole season. Only sampling lines/stations are regarded as larval samples.

\begin{tabular}{|l|cccccc|}
\hline Bioprov. & Species & $\begin{array}{l}\text { Sweep- } \\
\text { net }\end{array}$ & $\begin{array}{l}\text { Malaise- } \\
\text { traps }\end{array}$ & $\begin{array}{l}\text { Emerg. } \\
\text { traps }\end{array}$ & $\begin{array}{l}\text { Exuvíal } \\
\text { samples }\end{array}$ & $\begin{array}{l}\text { Larval } \\
\text { sites }\end{array}$ \\
\hline $\mathrm{Al}(\mathrm{A})$ & 192 & 22 & 19 & & & \\
$\mathrm{Ab}(\mathrm{V})$ & 510 & 794 & 78 & 37 & 15 & 310 \\
$\mathrm{~N}(\mathrm{U})$ & 442 & 134 & 42 & 12 & 14 & 187 \\
$\mathrm{Ka}(\mathrm{EK})$ & 378 & 163 & 3 & & 30 & 40 \\
$\mathrm{St}(\mathrm{St})$ & 467 & 149 & 25 & 10 & 3 & 114 \\
$\mathrm{Ta}(\mathrm{EH})$ & 523 & 365 & 44 & 20 & 52 & 388 \\
$\mathrm{Sa}(\mathrm{ES})$ & 414 & 137 & 2 & & 10 & 160 \\
$\mathrm{Kl}(\mathrm{LK})$ & 261 & 39 & & & 5 & 9 \\
Oa (EP) & 426 & 302 & 34 & 13 & 18 & 136 \\
$\mathrm{~Tb}(\mathrm{PH})$ & 481 & 241 & 20 & & 23 & 139 \\
$\mathrm{Sb}(\mathrm{PS})$ & 436 & 189 & 24 & & & 108 \\
$\mathrm{~Kb}(\mathrm{PK})$ & 452 & 147 & 33 & 3 & & 139 \\
Om (KP) & 415 & 159 & 5 & 4 & 16 & 136 \\
Ok (Kn) & 354 & 33 & 12 & & & 101 \\
Oba (OP) & 384 & 41 & 32 & & & 121 \\
Obb (PeP) & 411 & 33 & 32 & & 5 & 87 \\
Ks (Ks) & 459 & 42 & 65 & & & 319 \\
Lkoc (KiL) & 466 & 186 & 45 & & & 82 \\
Lkor (SoL) & 444 & 52 & 26 & 18 & & 78 \\
Le (EnL) & 494 & 137 & 55 & & 1 & 52 \\
Li (InL) & 553 & 471 & 36 & 4 & 1 & 96 \\
\hline Total & 781 & 3836 & 632 & 121 & 193 & 2802 \\
\hline & & & & & & \\
\hline
\end{tabular}




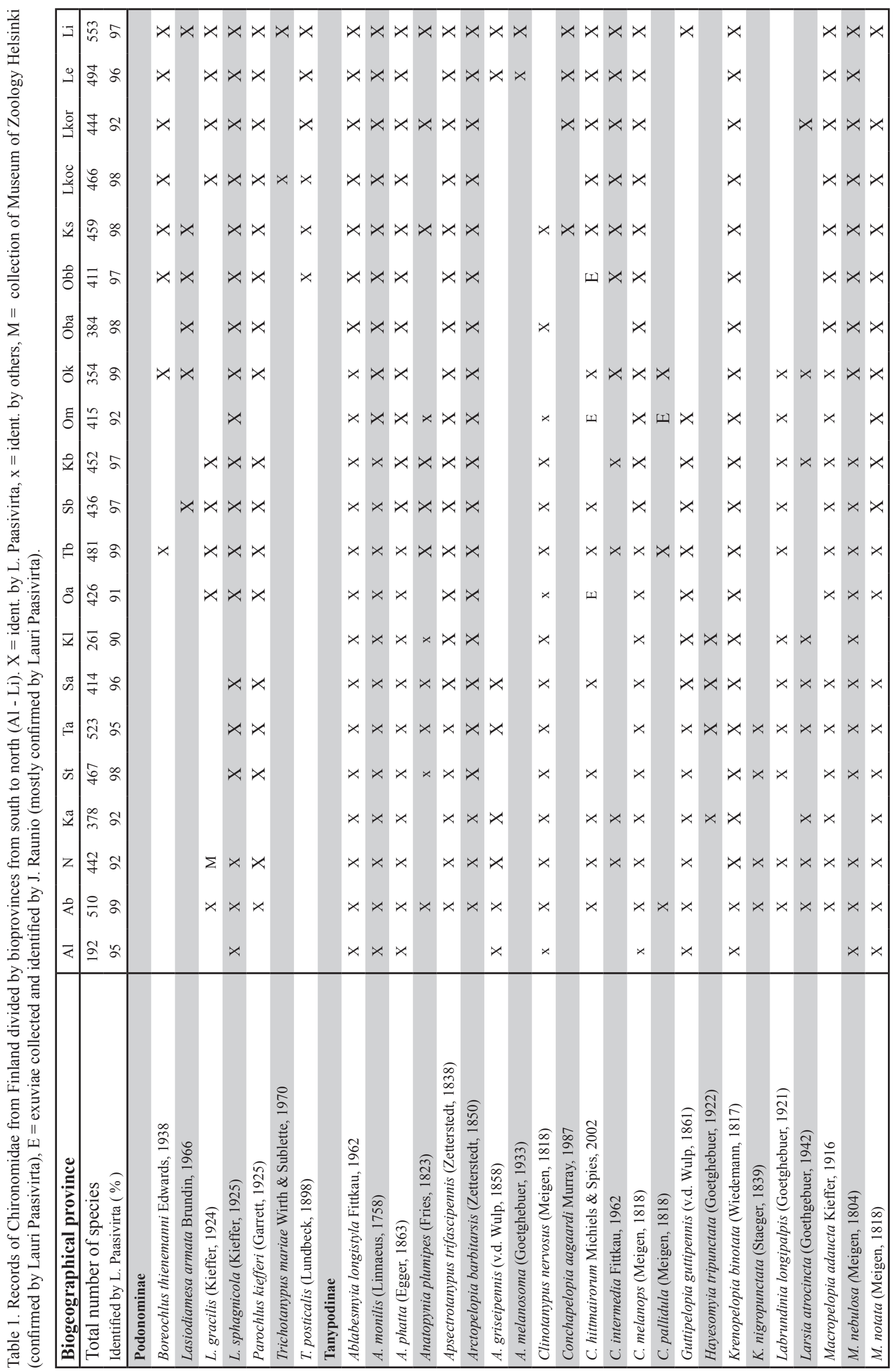




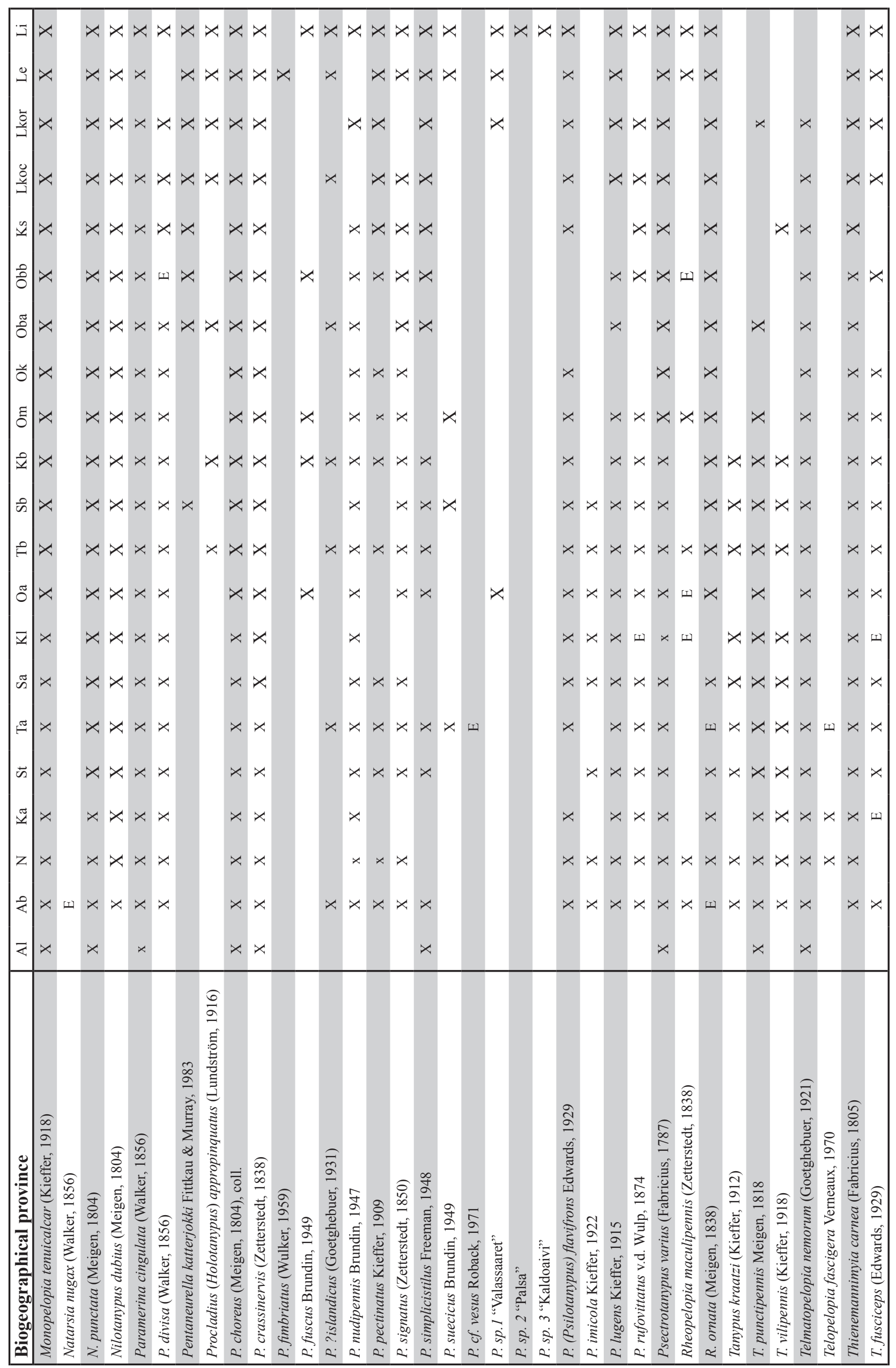




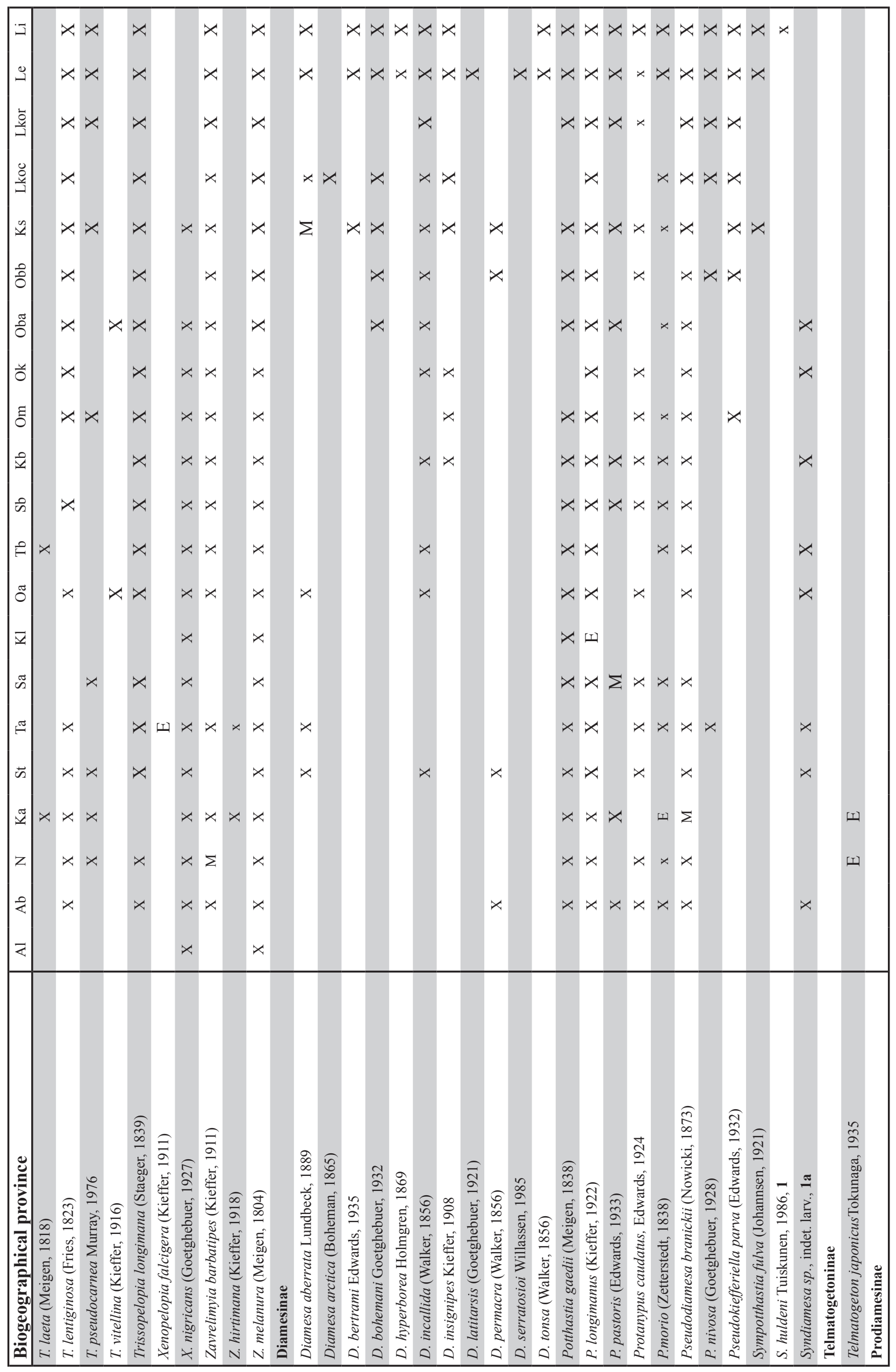




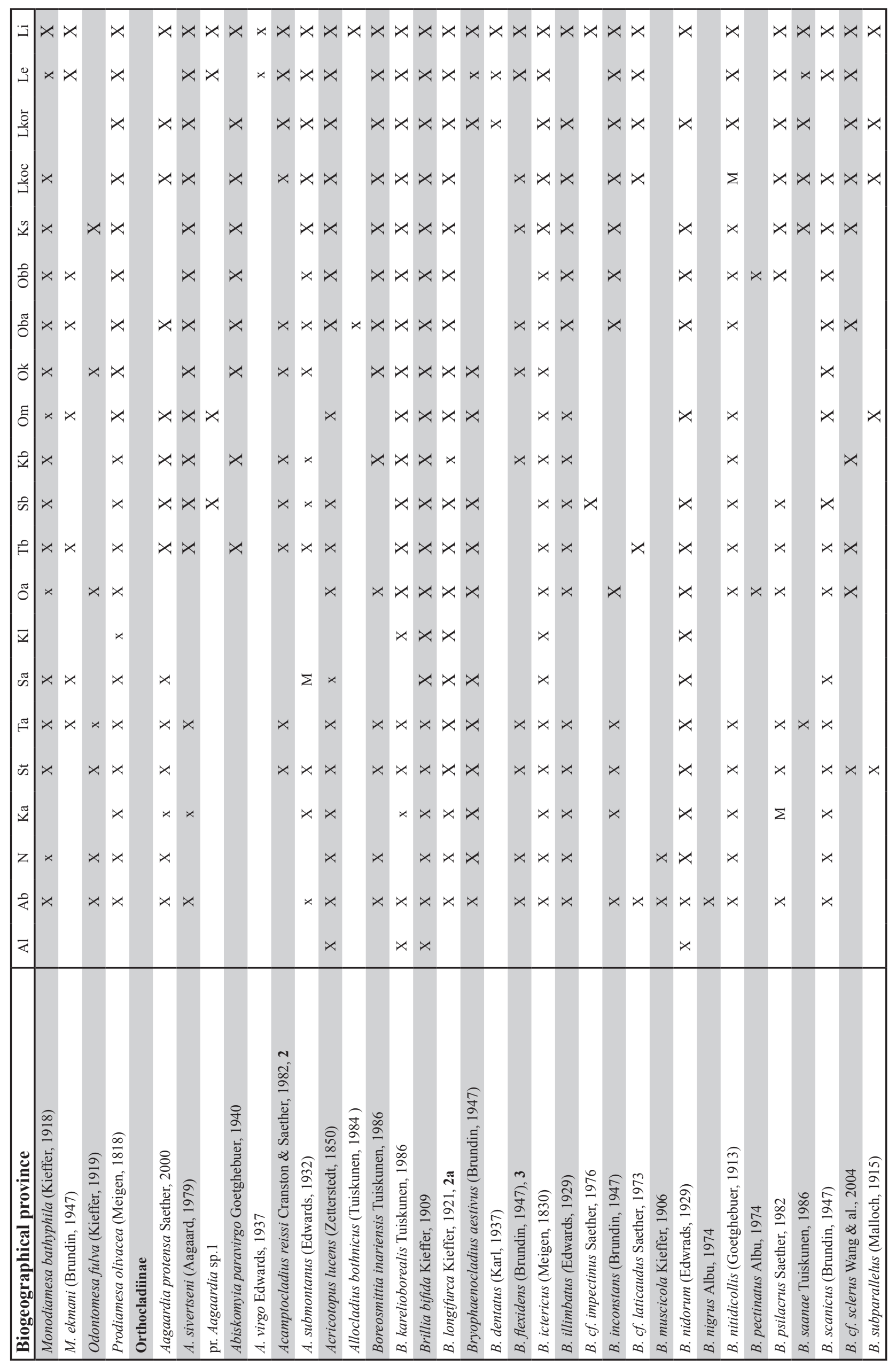




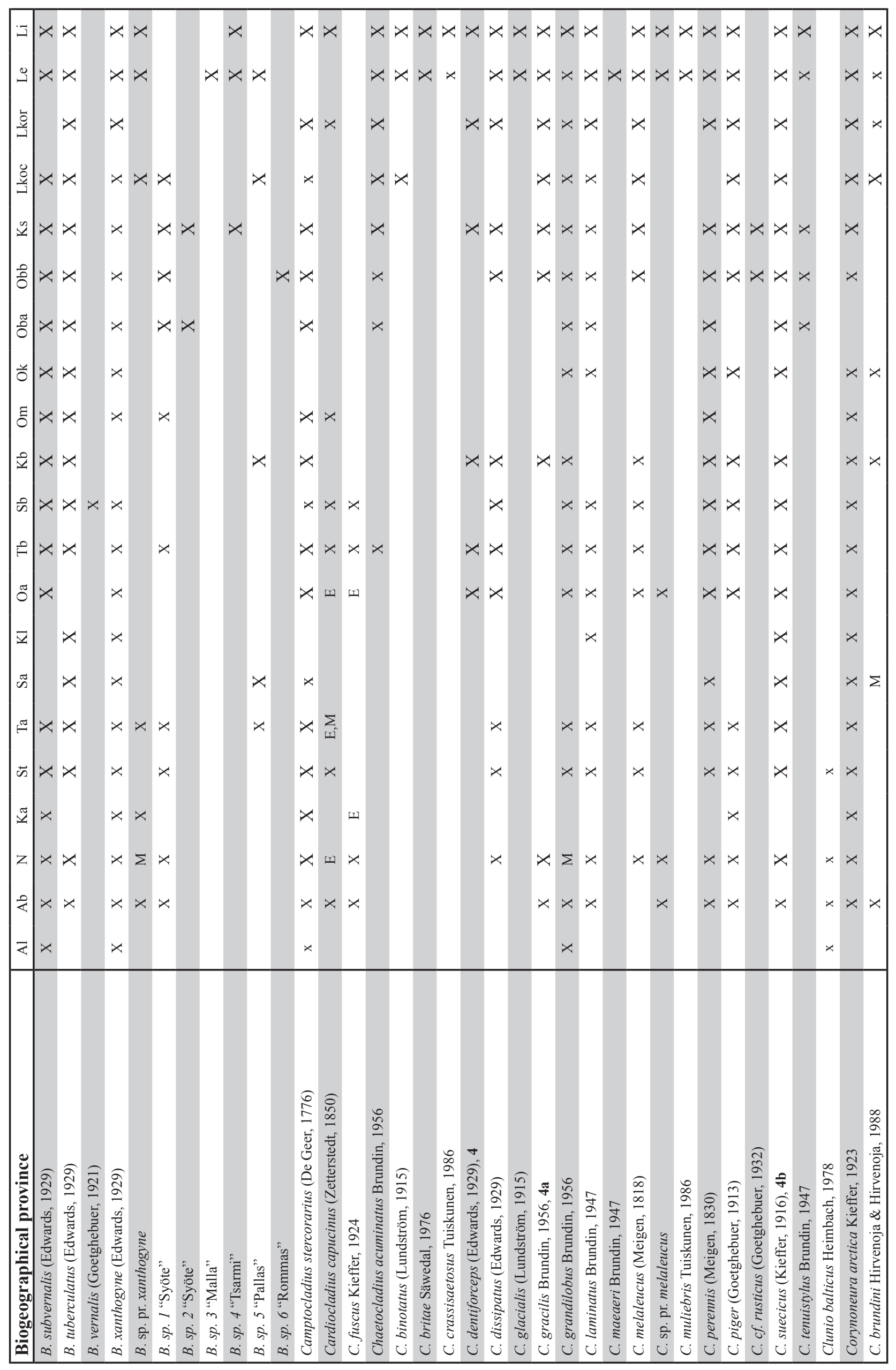




\begin{tabular}{|c|c|c|c|c|c|c|c|c|c|c|c|c|c|c|c|c|c|c|c|c|c|c|c|c|c|c|c|c|c|c|c|c|c|c|}
\hline ت & & $x$ & $x$ & & $x$ & $x$ & & & $x$ & $x$ & $x$ & $x$ & $x$ & & $x$ & $x$ & $x$ & $x$ & & $x$ & & $x \times$ & $<x$ & $\langle x$ & $x$ & $x>$ & $x \times$ & $x$ & $x$ & $x$ & $x$ & $x$ & $<>$ & $x$ \\
\hline ב & & $x$ & $x$ & & $x$ & $x$ & & $x$ & $x$ & $x$ & & $x$ & $x$ & $x$ & $x$ & & $x$ & $x$ & & $x>$ & & $x \times$ & $<x$ & $<x$ & $x$ & $x>$ & $x \times$ & $x$ & $x$ & & $x$ & & $x>$ & $x$ \\
\hline $\overrightarrow{\vec{y}}$ & & $x$ & $x$ & $x$ & $x$ & $x$ & & & $x$ & $x$ & & $x$ & $x$ & & $x$ & $x$ & $x$ & $x$ & & $x$ & & $x \times$ & $<x$ & $\Delta x$ & & $x>$ & $x \times$ & $x$ & $x$ & & $x$ & & & $x$ \\
\hline 8 & & $x$ & $x$ & $x$ & $x$ & $x$ & $x$ & & $x$ & $x$ & & $x$ & $x$ & & $x$ & & $x$ & $x$ & & $x$ & & $x=$ & $x x$ & $<x$ & & & $x$ & $x$ & $x$ & & $x$ & & & $x$ \\
\hline$v$ & & $x$ & $x$ & $x$ & $x$ & $x$ & $x$ & & $x$ & $x$ & & $x$ & & & $x$ & $x$ & $x$ & $x$ & & $x$ & & $x \times$ & $<x$ & $\angle x$ & & & $x \times$ & & $x$ & & $x$ & & $x>$ & $x$ \\
\hline है & & $x$ & $x$ & $x$ & $x$ & $x$ & 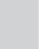 & & $x$ & $x$ & & $x$ & $x$ & & $x$ & $x$ & $x$ & $x$ & & & & $x$ & $<x$ & $<x$ & $x$ & & $x$ & & $x$ & & $x$ & & & \\
\hline ठే & $x$ & $x$ & $x$ & $x$ & & $x$ & 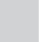 & & $x$ & $x$ & & $x$ & & & & $x$ & $x$ & $x$ & & & & $x \times$ & $4 x$ & $<x$ & 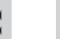 & & $x$ & & $x$ & & $x$ & & & $x$ \\
\hline ठै & & $x$ & $x$ & $x$ & $x$ & $x$ & & & $x$ & $x$ & & $x$ & & & & $x$ & $x$ & $x$ & & & & $x$ & $<x$ & $<x$ & & & $x$ & & $x$ & & $x$ & & & $\rtimes$ \\
\hline కే & $x$ & $x$ & $x$ & $x$ & $x$ & $x$ & $x$ & & $x$ & $x$ & & $x$ & & & & $x$ & $x$ & $x$ & & & & $x \times$ & $<x$ & $\langle x$ & $x$ & & $x$ & $x$ & $x$ & & $x$ & & & $x$ \\
\hline$\vec{\nabla}$ & $x$ & $x$ & & & $x$ & $x$ & $x$ & & $x$ & $x$ & & $x$ & $x$ & & & $x$ & $x$ & $x$ & & $x$ & & $x$ & $<x$ & $<x$ & $x$ & & $x$ & & $x$ & & $x$ & & & \\
\hline क & $x$ & $x$ & $x$ & $x$ & $x$ & $x$ & 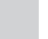 & & $x$ & $x$ & & $x$ & $x$ & & $x$ & $x$ & $x$ & 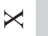 & & & & $x \times$ & $<x$ & $<x$ & & & $x$ & & $x$ & & $x$ & & & \\
\hline$\hat{F}$ & & $x$ & & $x$ & $x$ & $x$ & $x$ & & $x$ & $x$ & & $x$ & $x$ & & $x$ & $x$ & $x$ & $x$ & & $x$ & & $x \times$ & $4 x$ & $4 x$ & & & $x$ & & $x$ & & $x$ & & & \\
\hline రే & $x$ & $x$ & & $x$ & $x$ & $x$ & $x$ & & $x$ & $x$ & & $x$ & $x$ & & $x$ & $x$ & $x$ & $x$ & & & & $x \times$ & $x x$ & $4 x$ & & & $x$ & & $x$ & & $x$ & & & $x$ \\
\hline $\bar{v}$ & & $x$ & & $x$ & & & & & $x$ & & & $x$ & & & & $x$ & $x$ & $x$ & & & & $x x$ & $<x$ & $<x$ & $x$ & & $x$ & & $x$ & & $x$ & & & \\
\hline ஜ็ & $\Sigma$ & $x$ & $x$ & $x$ & $x$ & $x$ & $x$ & & $x$ & $x$ & & $x$ & . & & $x$ & $x$ & $x$ & $x$ & & $x$ & & $x$ & $<x$ & $4 x$ & $x$ & & $x$ & & $x$ & $x$ & $x$ & & & \\
\hline 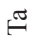 & $x$ & $x$ & $x$ & $x$ & $x$ & $x$ & $x$ & & $x$ & $x$ & & $x$ & $x$ & & $x$ & $x$ & $x$ & $x$ & & $x$ & & $x \times$ & $4 x$ & $<x$ & $x$ & $x$ & $x$ & & $x$ & & $x$ & & & $x$ \\
\hline$\vec{\omega}$ & $x$ & $x$ & & $x$ & $x$ & $x$ & $x$ & & $x$ & $x$ & & $x$ & $x$ & & $x$ & $x$ & $x$ & $x$ & $x$ & & & $x$ & $x$ & $<x$ & $x$ & & $x$ & & $x$ & & $x$ & & & \\
\hline$\tilde{\simeq}$ & $x$ & $x$ & $x$ & $x$ & $x$ & $x$ & 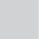 & & $x$ & $x$ & & $x$ & . & & & $x$ & $x$ & $x$ & & & & $x$ & $x$ & $<x$ & 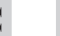 & & $x$ & & $x$ & & $x$ & & & \\
\hline z & $x$ & $x$ & & $x$ & $x$ & $x$ & 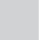 & & $x$ & $x$ & & $x$ & $x$ & & & $x$ & $x$ & $x$ & $\star$ & & & $x$ & $4 x$ & $<x$ & $x$ & & & & $\star$ & & $x$ & & & \\
\hline 운 & $x$ & $x$ & $x$ & $x$ & $x$ & $x$ & $x$ & & $x$ & $x$ & & $x$ & $x$ & & & $x$ & $x$ & $x$ & $x$ & 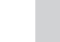 & & $x \times$ & $<x$ & $<x$ & $x$ & . & $x$ & & $x$ & & $x$ & & & \\
\hline を & & $x$ & & & $x$ & $x$ & & & $x$ & & & $x$ & & & & $x$ & & $x$ & $x$ & & & & $x$ & & & & & & & & & & & \\
\hline 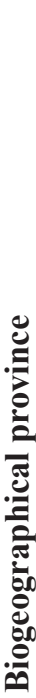 & 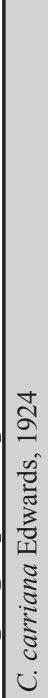 & 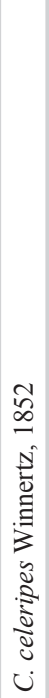 & 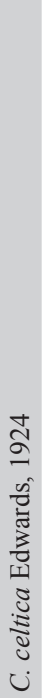 & 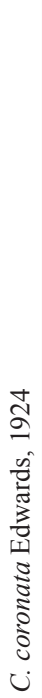 & 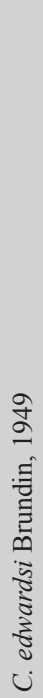 & 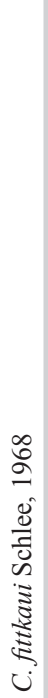 & 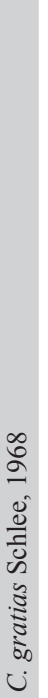 & 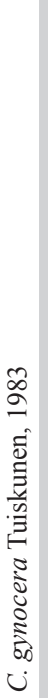 & 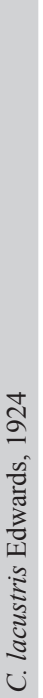 & 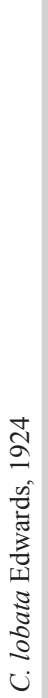 & 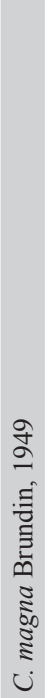 & 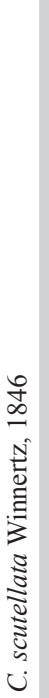 & 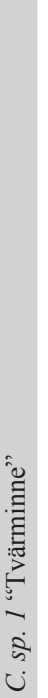 & $\begin{array}{l}\mathcal{N} \\
\dot{m} \\
\dot{m} \\
u\end{array}$ & 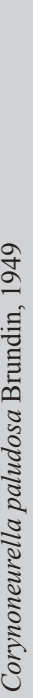 & 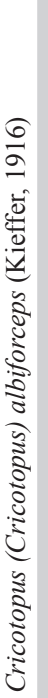 & 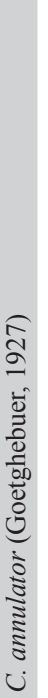 & 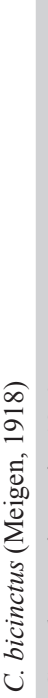 & 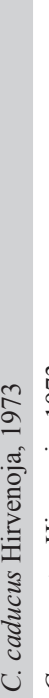 & 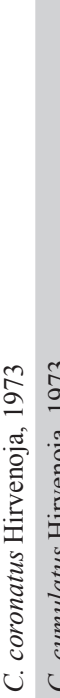 & 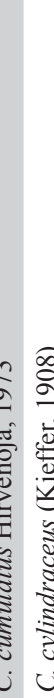 & 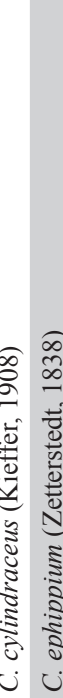 & 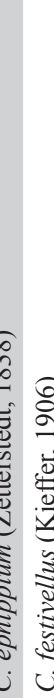 & 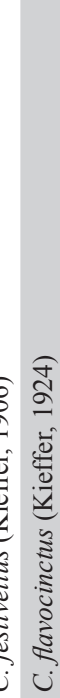 & 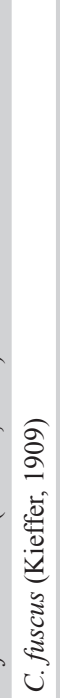 & 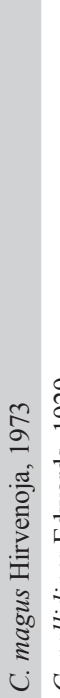 & 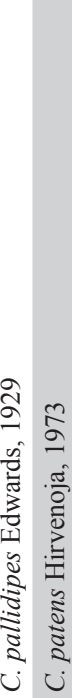 & 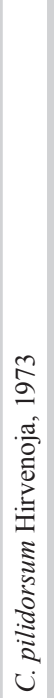 & 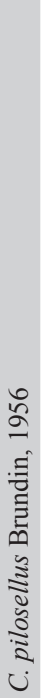 & 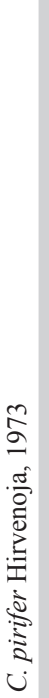 & 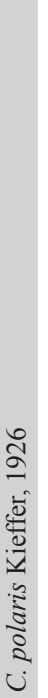 & 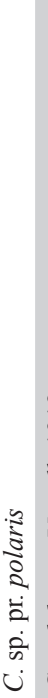 & 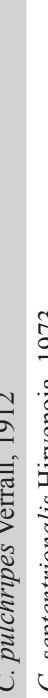 & 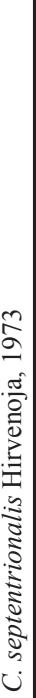 \\
\hline
\end{tabular}




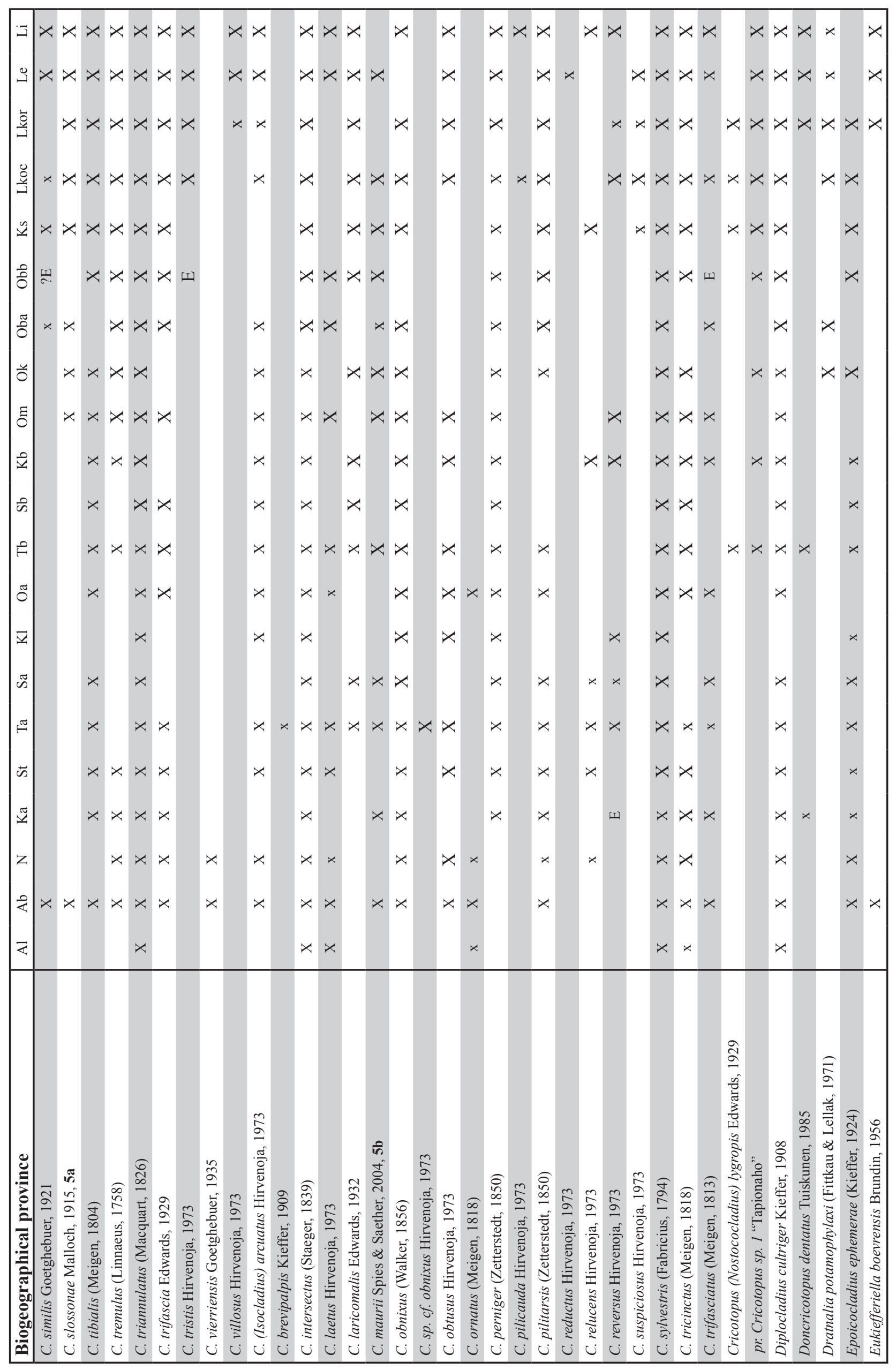




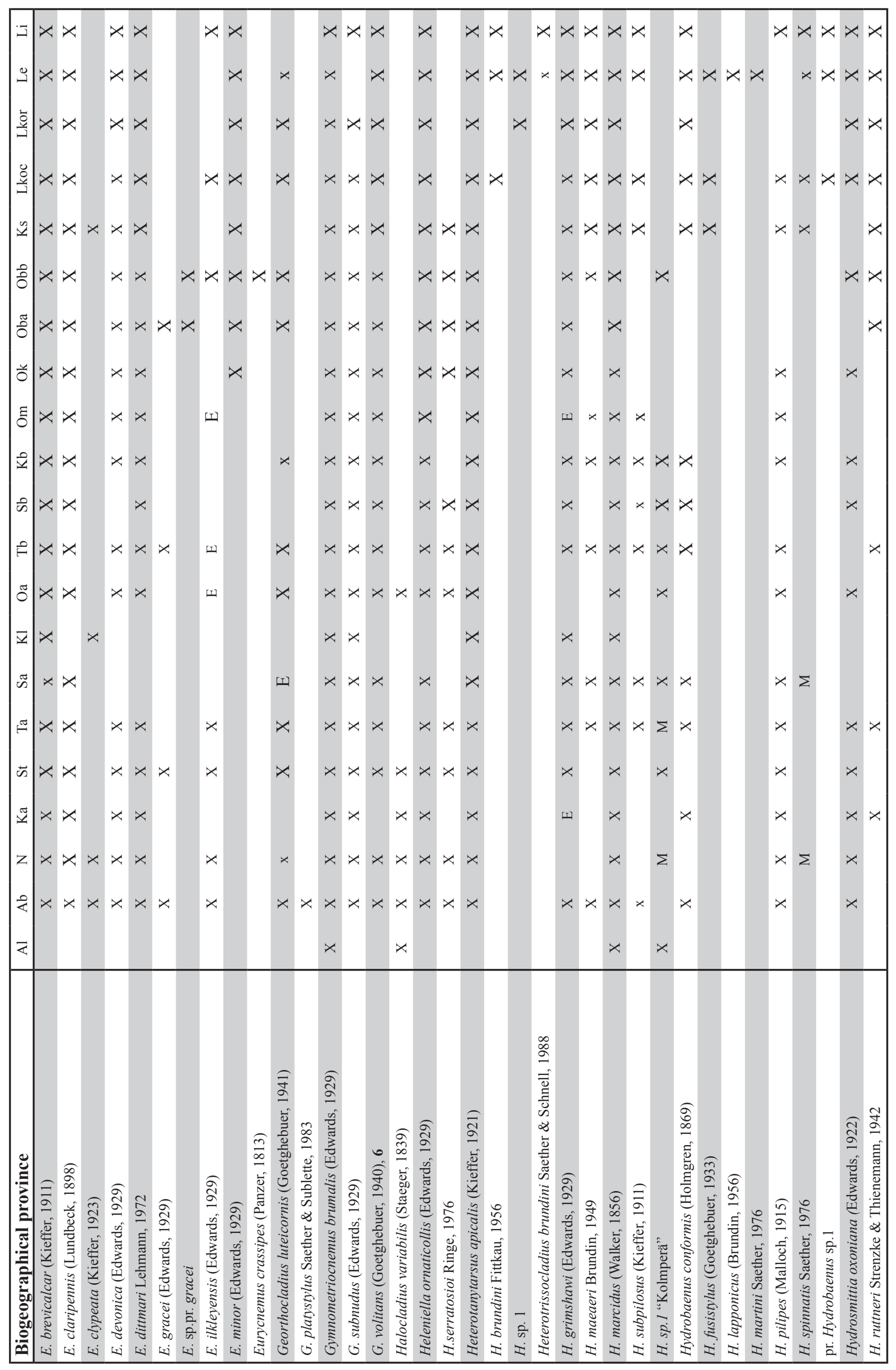




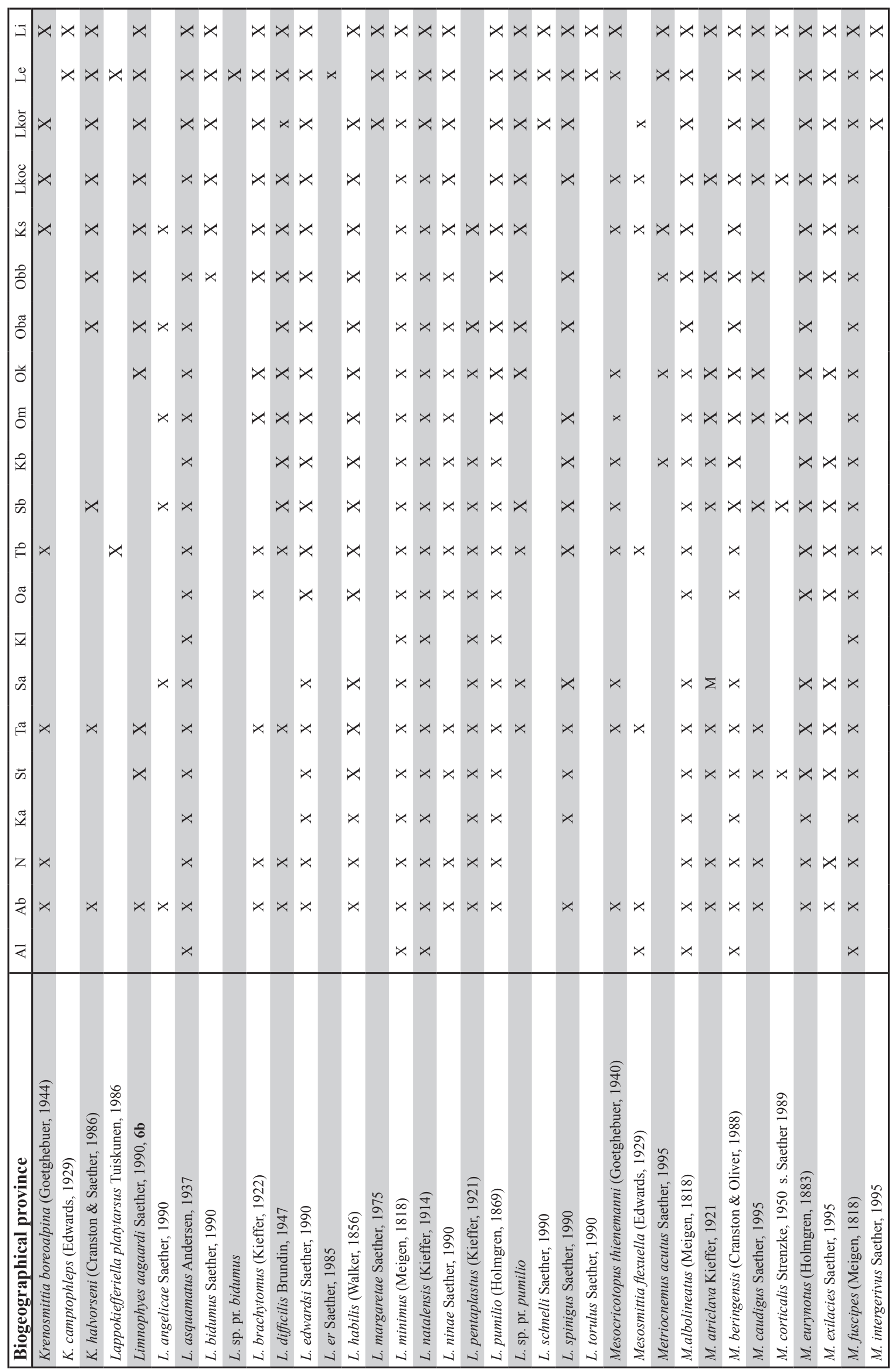




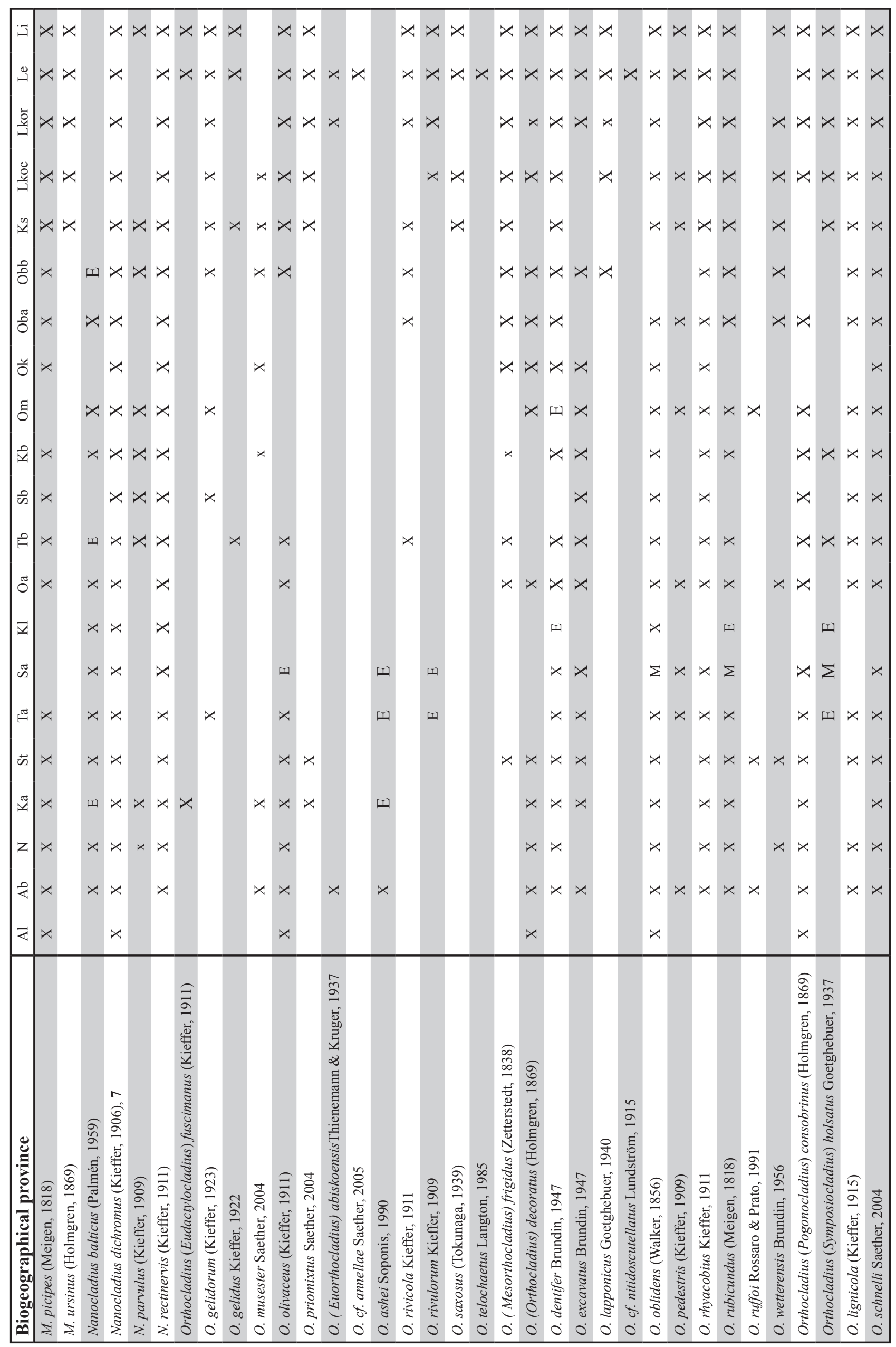




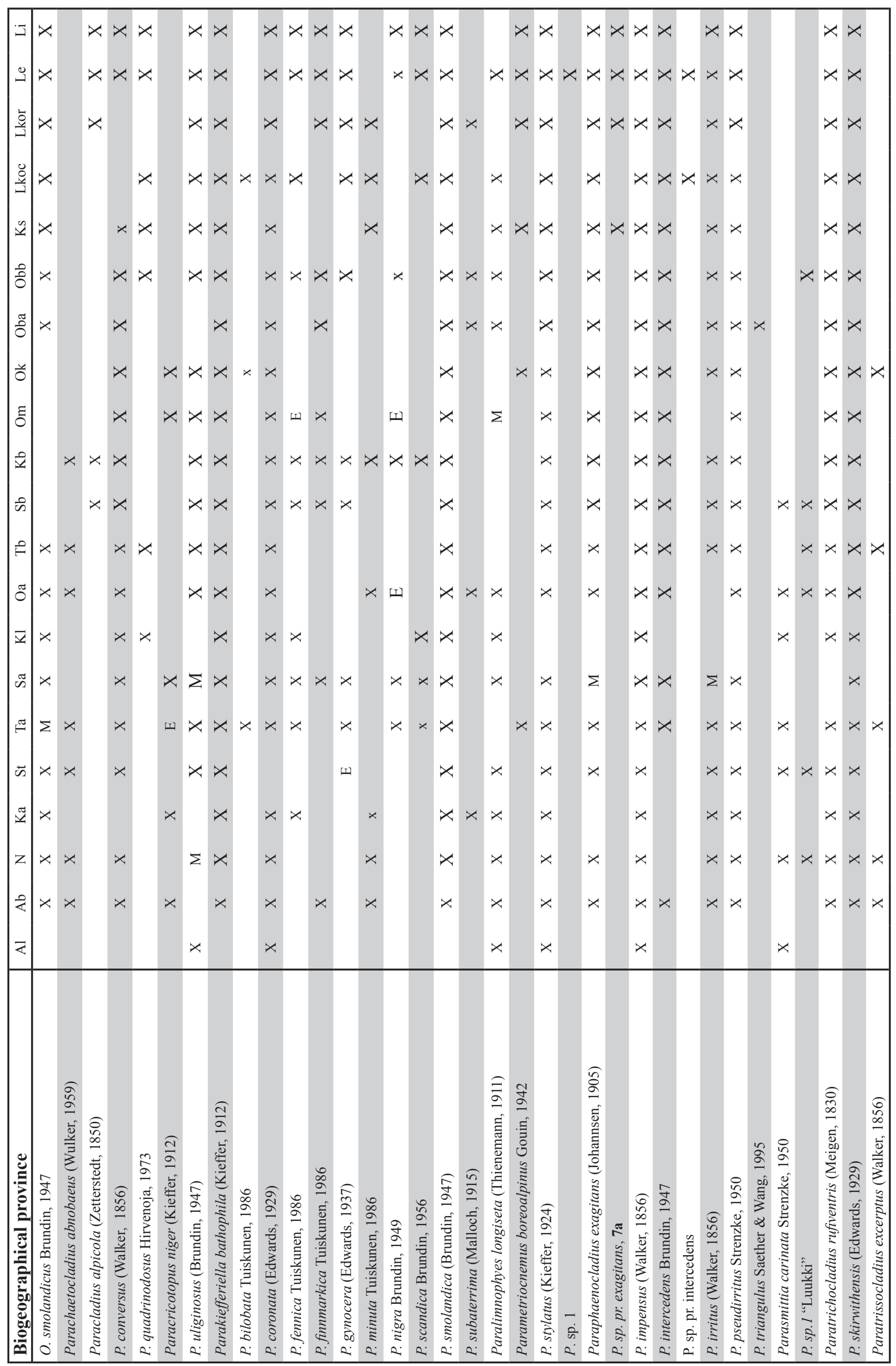




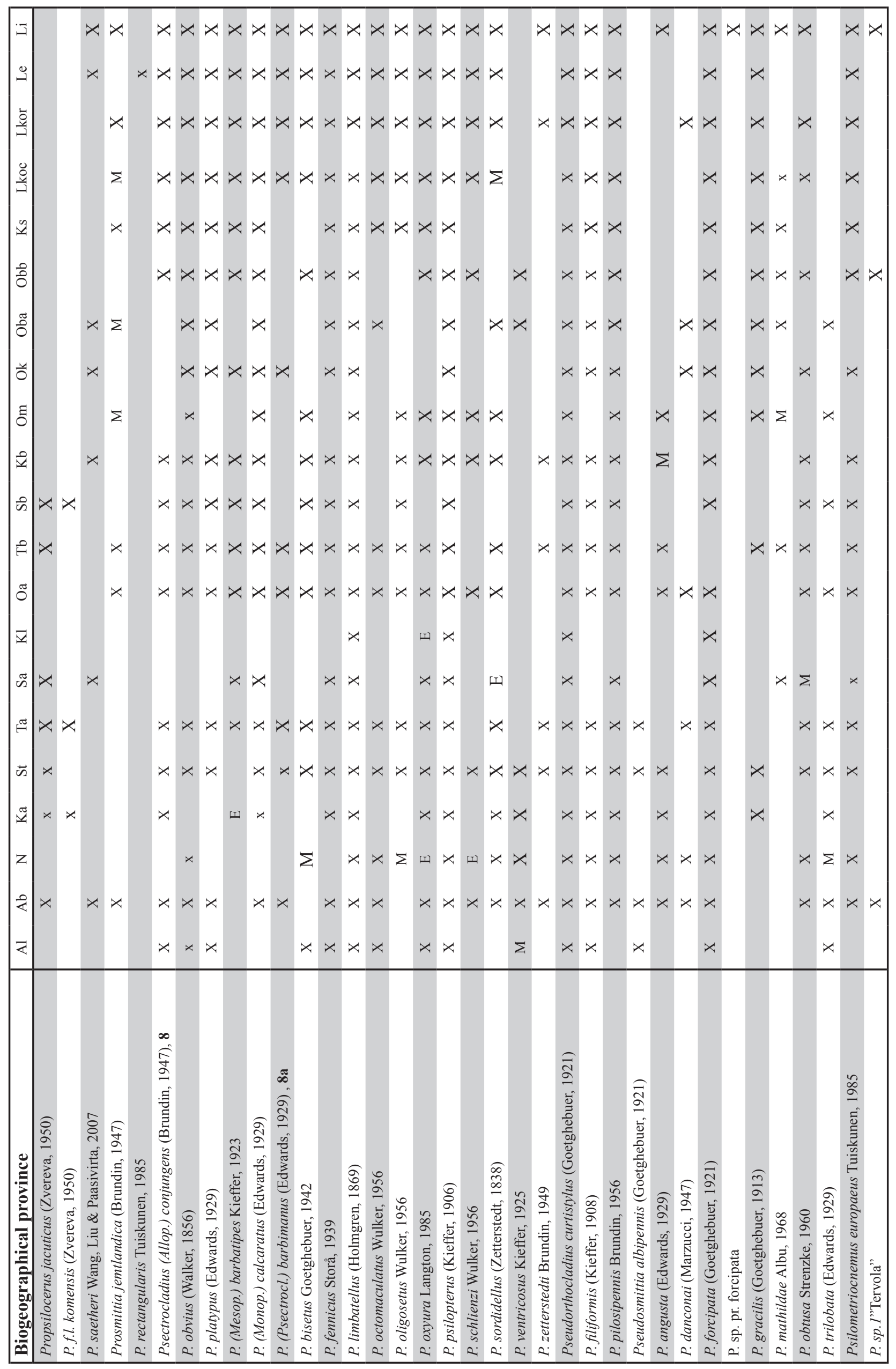




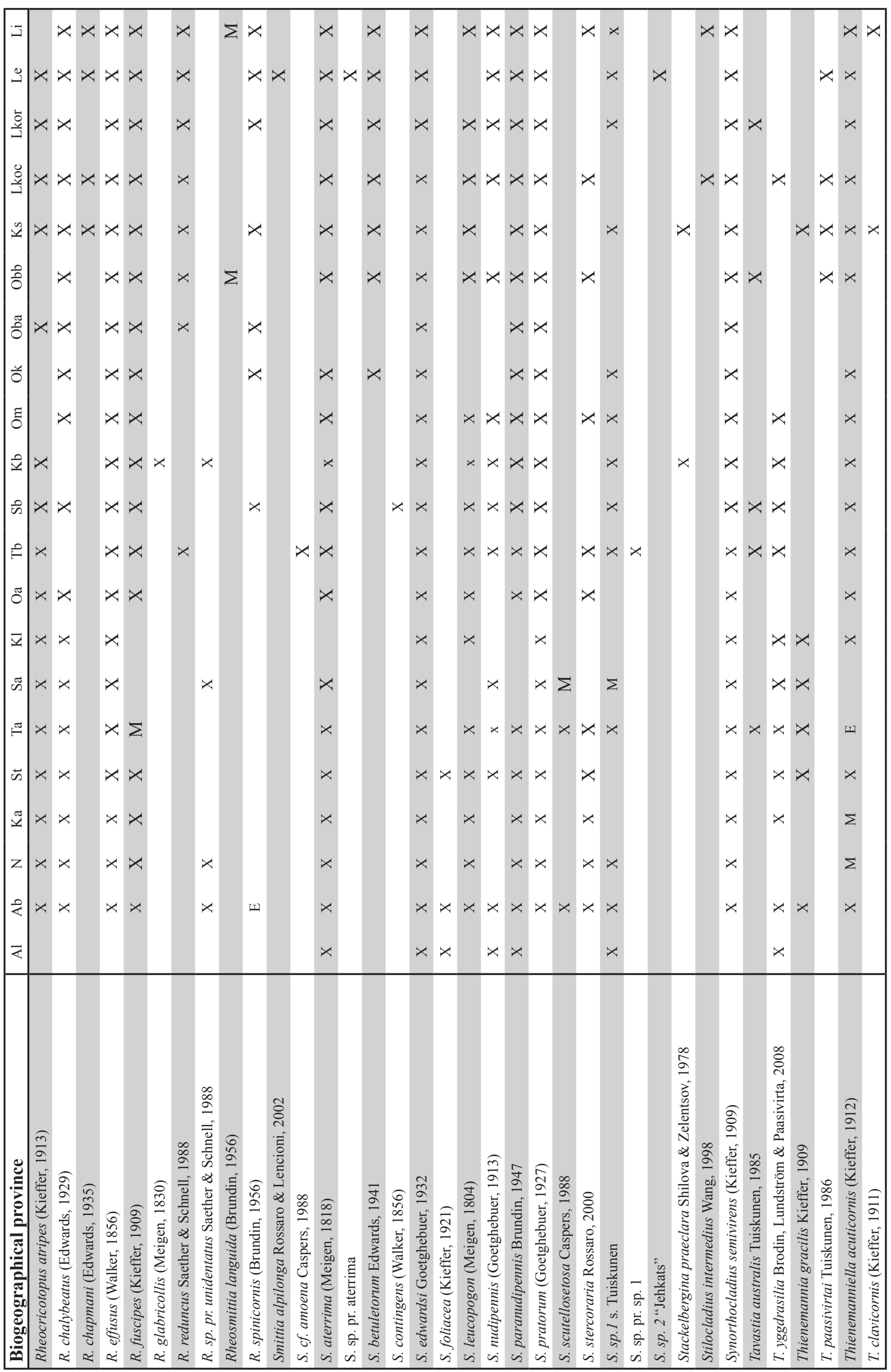




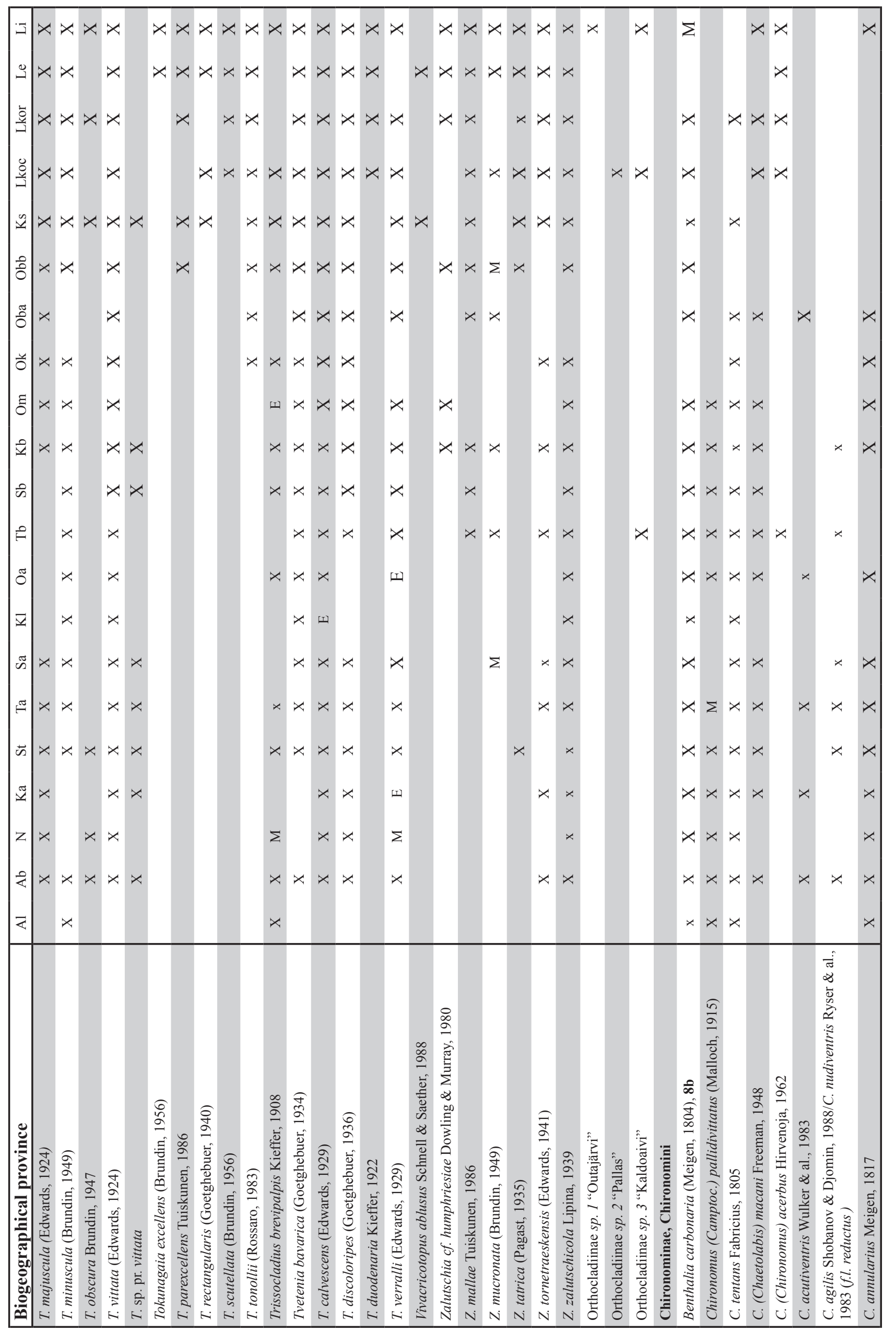




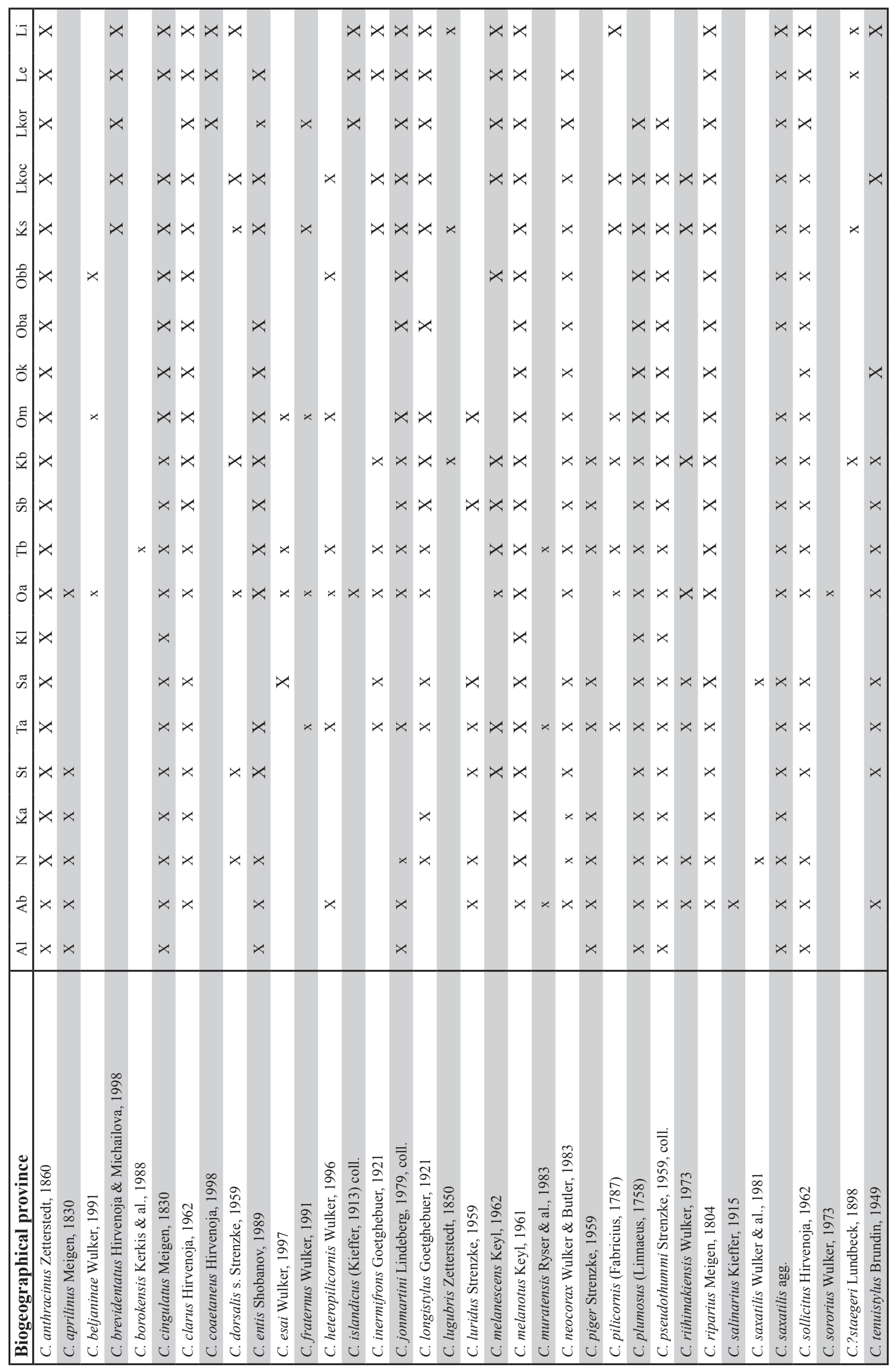




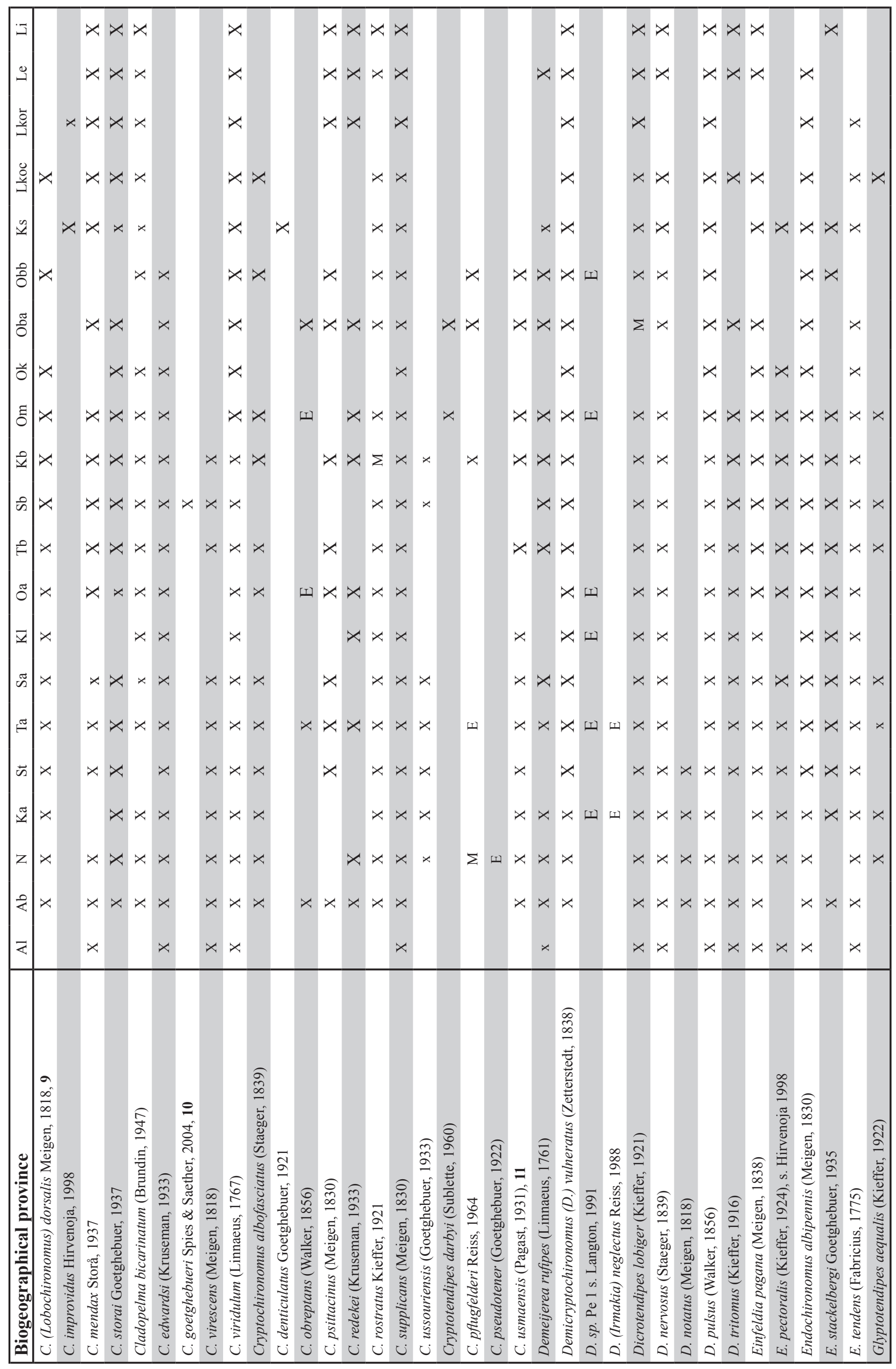




\begin{tabular}{|c|c|c|c|c|c|c|c|c|c|c|c|c|c|c|c|c|c|c|c|c|c|c|c|c|c|c|c|c|c|c|c|c|c|c|c|}
\hline ت & & & $x$ & & & & $x$ & $x$ & & & & & & & $x$ & & & & $x$ & $x$ & $X$ & $x>$ & $x$ & & $x$ & $\angle X$ & $x$ & $x$ & $x$ & $x$ & $x$ & & & & $x$ \\
\hline 9 & & & & & & & $x$ & $x$ & & & & & & & $x$ & & & & $x$ & $x$ & & $x>$ & $x$ & & $x$ & $<x$ & $x$ & $x$ & $x$ & $x$ & $x$ & $x$ & & & $x$ \\
\hline 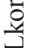 & & & $x$ & & & & $x$ & $x$ & & & & & & & $x$ & & & $x$ & $x$ & $x$ & & $x>$ & $x$ & & $x$ & $\angle x$ & & & $x$ & $x$ & $x$ & & $x$ & $x$ & \\
\hline$\stackrel{0}{y}$ & & & $X$ & & & & $x$ & $x$ & $x$ & & & $x$ & & & $x$ & & & & 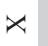 & $x$ & & $\times>$ & $x$ & & $x$ & $\langle x$ & $x$ & & $x$ & & $x$ & & $x$ & & $x$ \\
\hline$\ddot{v}$ & & & $x$ & & & & $x$ & $x$ & $x$ & & & $x$ & & & $x$ & & & & $x$ & $x$ & & $x>$ & $x$ & & $x$ & $<x$ & $x$ & & $x$ & $\Sigma$ & $x$ & & & $x$ & \\
\hline ०ै & & & $x$ & & & & $x$ & $x$ & $x$ & & $x$ & II & & & $x$ & & & $x$ & $x$ & $x$ & $x$ & & $x$ & & & $x$ & & & $x$ & $x$ & $x$ & & & $x$ & \\
\hline อొ & & & $x$ & & & & $x$ & $x$ & $x$ & & $x$ & & & & & & & & $x$ & $x$ & $x$ & & $x$ & & & $x$ & & & $x$ & $x$ & $x$ & & $x$ & & $x$ \\
\hline ठै & & & $x$ & $x$ & & & $x$ & $x$ & & & $x$ & & & & $x$ & & $x$ & & $x$ & $x$ & & & $x$ & $>$ & $x>$ & $\angle X$ & & & & & $x$ & & & & $x$ \\
\hline छี & & & $x$ & $x$ & & $x$ & $x$ & $x$ & $x$ & & $x$ & I & $x$ & & $x$ & & $x$ & $x$ & & $x$ & $x$ & & $x$ & & $x \times$ & $<x$ & $x$ & & $x$ & 工工 & $x$ & $x$ & $x$ & & $x$ \\
\hline$\vec{\Delta}$ & & & $x$ & & & $x$ & $x$ & $x$ & $x$ & & $x$ & $x$ & & $x$ & $x$ & & & $x$ & $x$ & $x$ & $x$ & $x>$ & $x$ & $x>$ & $x \times$ & $<x$ & & & $x$ & $x$ & $x$ & $x$ & $x$ & $x$ & \\
\hline ฉ & & & $x$ & $x$ & & $x$ & $x$ & $x$ & $x$ & $x$ & $x$ & & $x$ & & $x$ & & $x$ & $x$ & $x$ & $x$ & $x$ & $>$ & $x$ & $x>$ & $x \times$ & $4 x$ & $x$ & $x$ & $x$ & $x$ & $x$ & $x$ & $x$ & $x$ & \\
\hline F & & & $x$ & $x$ & & $X$ & $x$ & $x$ & $x$ & & $x$ & $x$ & $x$ & & $x$ & & & $x$ & $x$ & $x$ & $x$ & $x>$ & $x$ & $x>$ & $x \times$ & $\langle x$ & $x$ & & $x$ & $x$ & $x$ & $x$ & $x$ & & $x$ \\
\hline రొ & $x$ & & $x$ & $x$ & $x$ & $X$ & $x$ & $x$ & 떠 & & $x$ & 더 & $x$ & & $\rtimes$ & & & $x$ & & $x$ & $x$ & $>$ & $x$ & & $x$ & $x$ & & & $x$ & $x$ & $x$ & $x$ & $x$ & & $x$ \\
\hline $\bar{v}$ & & & $x$ & $x$ & $x$ & $x$ & $x$ & $x$ & . & & $x$ & 디 & & & $x$ & & & $x$ & $x$ & $x$ & & & $x$ & & $x>$ & $\angle X$ & $x$ & & & $x$ & $x$ & $x$ & $x$ & $x$ & \\
\hline$\tilde{\mathscr{n}}$ & & & $x$ & & $x$ & & $x$ & $x$ & $x$ & & $x$ & $x$ & $x$ & & $x$ & & & $x$ & $x$ & $x$ & $x$ & $\Sigma>$ & $x$ & $x>$ & $x \times$ & $<x$ & $x$ & & & $x$ & $x$ & $x$ & $x$ & $x$ & \\
\hline [) & 디 & & $x$ & $x$ & $x$ & $x$ & $x$ & $x$ & $x$ & $x$ & $x$ & 띠 & 피 & & $x$ & $x$ & $x$ & $x$ & $x$ & $x$ & $x$ & $>$ & $x$ & $x>$ & $x x$ & $<x$ & $x$ & & $x$ & $x$ & $x$ & $x$ & $x$ & $x$ & \\
\hline$\ddot{n}$ & $x$ & & $x$ & $x$ & $x$ & $x$ & $x$ & $x$ & $x$ & & $x$ & $x$ & $x$ & & $x$ & & & $x$ & $x$ & $x$ & & & $x$ & & $x x$ & $<x$ & $x$ & & $x$ & $x$ & $x$ & $x$ & $x$ & $x$ & \\
\hline$\tilde{\Xi}$ & & & $x$ & $x$ & $x$ & & $x$ & $x$ & $x$ & $x$ & $x$ & $x$ & $x$ & 디 & $x$ & & & $x$ & $x$ & $x$ & & $>$ & $x$ & $\Sigma>$ & $x \times$ & $<x$ & $x$ & & $x$ & $x$ & $x$ & $x$ & $x$ & $x$ & \\
\hline z & $x$ & & $x$ & $x$ & $x$ & $x$ & $x$ & $x$ & & $x$ & $x$ & $\Sigma$ & $x$ & & $x$ & & $x$ & $x$ & 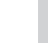 & $x$ & $x$ & $\Sigma>$ & $x$ & $\Sigma>$ & $x \times$ & $<x$ & $x$ & & $x$ & $x$ & $x$ & $x$ & $x$ & $x$ & \\
\hline \& & $x$ & $x$ & $x$ & $x$ & $x$ & $x$ & $x$ & $x$ & $x$ & $x$ & $x$ & & $x$ & I & $x$ & & & $x$ & $x$ & $x$ & $x$ & 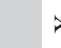 & $x$ & $x>$ & $x x$ & $<x$ & $x$ & & $x$ & $x$ & $x$ & $x$ & $x$ & $x$ & $x$ \\
\hline « & $x$ & & $x$ & & & & & $x$ & & & $x$ & & $x$ & & & & & $x$ & & $x$ & & & $x$ & & & $x$ & & & & & $x$ & & & & $x$ \\
\hline 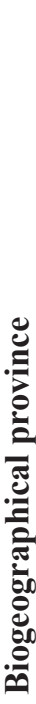 & 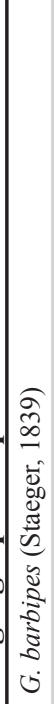 & 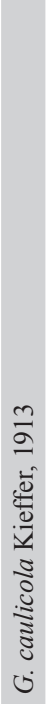 & 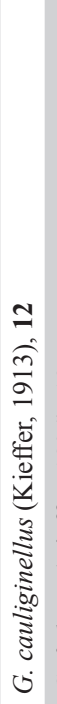 & 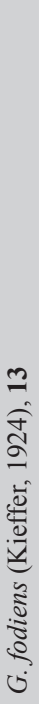 & 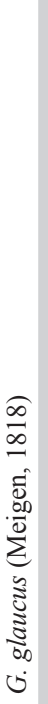 & 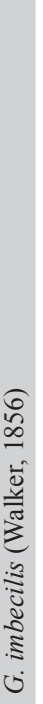 & 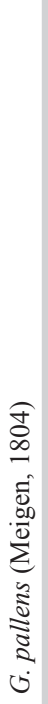 & 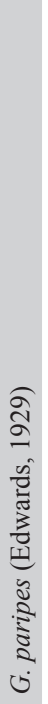 & 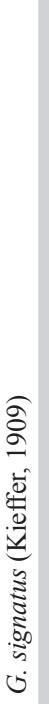 & 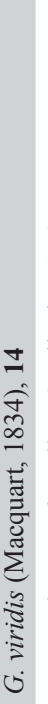 & 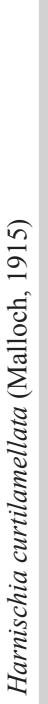 & 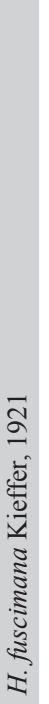 & 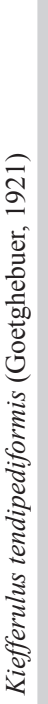 & 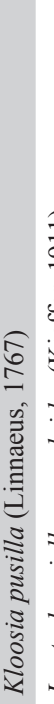 & 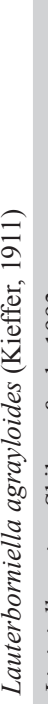 & 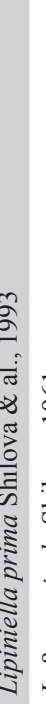 & 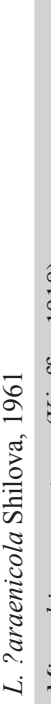 & 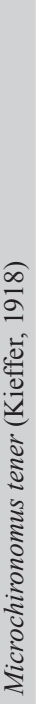 & 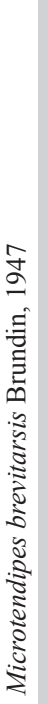 & 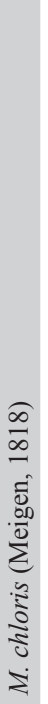 & 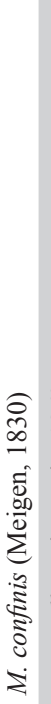 & 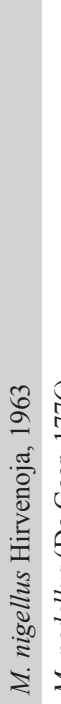 & 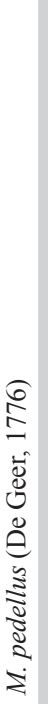 & 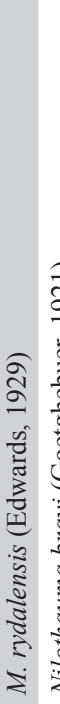 & 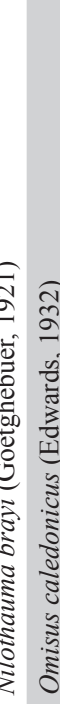 & 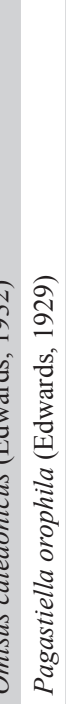 & 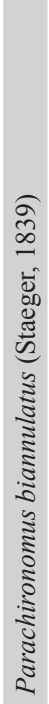 & 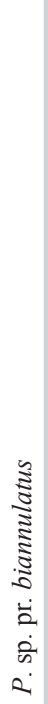 & 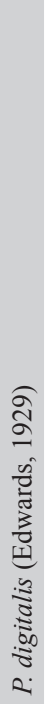 & 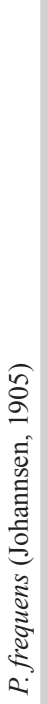 & 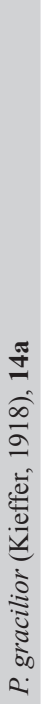 & 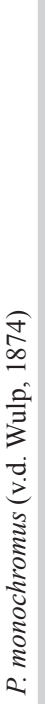 & 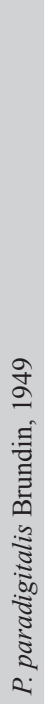 & 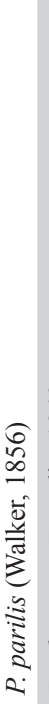 & 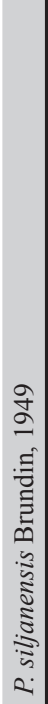 \\
\hline
\end{tabular}




\begin{tabular}{|c|c|c|c|c|c|c|c|c|c|c|c|c|c|c|c|c|c|c|c|c|c|c|c|c|c|c|c|c|c|c|c|c|c|c|}
\hline$\Xi$ & $x$ & & $x$ & $x$ & & $x$ & $x$ & $x$ & $x$ & & & $x$ & $x$ & $x$ & & $x$ & $x$ & $x$ & $x$ & & $x$ & & $x$ & $x$ & $x$ & $x$ & $x$ & $x$ & $x \times$ & & $x$ & $x$ & & $x x$ \\
\hline בּَ & $x$ & & $x$ & $x$ & & & $x$ & & $x$ & $x$ & & & $x$ & $x$ & & $x$ & $x$ & $x$ & $x$ & & $x$ & & $x$ & & & & $x$ & & $x$ & $x$ & $x$ & $x$ & & $x \times$ \\
\hline 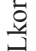 & $x$ & & $x$ & $x$ & & $x$ & & $x$ & $x$ & & & & $x$ & $x$ & & $x$ & $x$ & $x$ & $x$ & & $x$ & & & & $x$ & $x$ & $x$ & $x$ & $\chi x$ & $x$ & & $x$ & & $x$ \\
\hline 当 & $x$ & & $x$ & $x$ & & $x$ & & $x$ & $x$ & & & & $x$ & $x$ & & $x$ & $x$ & $x$ & $x$ & & $x$ & $x y$ & $x$ & & $x$ & $x$ & $x$ & $x$ & $x \times$ & & $x$ & $x$ & & $x$ \\
\hline$\tilde{v}$ & $x$ & $x$ & & $x$ & & $x$ & & $x$ & & $x$ & & $x$ & $x$ & $x$ & & $x$ & $x$ & $x$ & $x$ & & $x$ & & & & $x$ & $x$ & & $x x$ & $x \times$ & & $x$ & $x$ & $x>$ & $x$ \\
\hline है & & & & $x$ & & $x$ & & $x$ & & & $x$ & $x$ & $x$ & $x$ & & $x$ & $x$ & $x$ & $x$ & & $x$ & & $x$ & $x$ & $x$ & $x$ & $x$ & $x$ & $x \times$ & $x$ & $x$ & & & $x$ \\
\hline ठ̃ & & $x$ & $x$ & $x$ & $x$ & $x$ & $x$ & $x$ & & $x$ & & $x$ & $x$ & $x$ & & $x$ & $x$ & $x$ & $x$ & & $x$ & & $x$ & & $x$ & & & $x$ & $x \times$ & $x$ & $x$ & & & $x$ \\
\hline ö & $x$ & & & $x$ & & $x$ & $x$ & $x$ & & $x$ & & $x$ & $x$ & $x$ & & $x$ & $x$ & $x$ & $x$ & & $x$ & & $x$ & & $x$ & $x$ & $x$ & $x$ & $x \times$ & & $x$ & $x$ & $x>$ & $x$ \\
\hline కี & & $x$ & & $x$ & & $x$ & & $x$ & $\rtimes$ & & & $x$ & $x$ & $x$ & & $x$ & $x$ & $x$ & $x$ & & $x$ & & $x$ & $x$ & $x$ & $x$ & $x$ & $x x$ & $x<$ & & $x$ & $x$ & $x>$ & $x$ \\
\hline$\vec{v}$ & $x$ & $x$ & $x$ & $x$ & & $x$ & & $x$ & $x$ & & & $x$ & $x$ & $x$ & & $x$ & $x$ & $x$ & $x$ & $x$ & $x$ & & $x$ & $x$ & $x$ & $x$ & & $x$ & $x \times$ & & $x$ & $x$ & & $x x$ \\
\hline$\vec{n}$ & & & $x$ & $x$ & & $x$ & $x$ & $x$ & $\rtimes$ & & & $x$ & $x$ & $x$ & & $x$ & $x$ & $x$ & $x$ & & $x$ & $x$ & $x$ & & $x$ & $x$ & $x$ & $x$ & $x \times$ & & $x$ & $x$ & $x>$ & $x$ \\
\hline 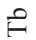 & & $x$ & & $x$ & & $x$ & & $x$ & $x$ & & & $x$ & $x$ & $x$ & & $x$ & $x$ & $x$ & $x$ & $x$ & $x$ & $x$ & $x$ & & $x$ & $x$ & $x$ & $x$ & $x \times$ & & $x$ & $x$ & 피 > & $x$ \\
\hline రే & & $x$ & $x$ & $x$ & & $x$ & & 巧 & & & & $x$ & $x$ & $x$ & & $x$ & $x$ & $x$ & $x$ & & $x$ & & $x$ & $\star$ & $x$ & $x$ & $x$ & $x$ & $x \times$ & & $x$ & & & $x$ \\
\hline $\bar{v}$ & & & & & & [工] & & $x$ & & & & $x$ & & & & $x$ & $x$ & $x$ & $x$ & & & $x ;$ & $x$ & & $x$ & $x$ & & $x$ & $x \times$ & & $x$ & & & $x$ \\
\hline ஜ็ & $\Sigma$ & & & $x$ & & $x$ & & $x$ & $x$ & & & $x$ & $x$ & $x$ & & $x$ & $x$ & $x$ & $x$ & & & $\Sigma$ & & $x$ & $x$ & $x$ & $x$ & $x$ & $x \times$ & $x$ & $x$ & & $x>$ & $x$ \\
\hline$\pi$ & $x$ & $x$ & $x$ & $x$ & $x$ & $x$ & & $x$ & $x$ & $x$ & & $x$ & $x$ & $x$ & & $x$ & $x$ & $x$ & $x$ & $x$ & $x$ & $x ;$ & $x$ & $x$ & $x$ & $x$ & $x$ & $x$ & $x \times$ & $x$ & $x$ & $x$ & $x>$ & $x$ \\
\hline$\vec{\omega}$ & $x$ & $x$ & $x$ & $x$ & & $x$ & & $x$ & $x$ & & & $x$ & $x$ & $x$ & $x$ & $x$ & $x$ & $x$ & $x$ & $x$ & $x$ & $x$ & . & & $x$ & $x$ & $x$ & $x \times$ & $<x$ & & $x$ & 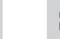 & $x>$ & $x x$ \\
\hline$\tilde{\check{u}}$ & & & $x$ & $x$ & & $x$ & & $x$ & & & & $x$ & $x$ & $x$ & & $x$ & $x$ & $x$ & $x$ & . & $x$ & i & $x$ & & $x$ & $x$ & & x & $x \times$ & & $x$ & $x$ & & $x$ \\
\hline z & & $\Sigma$ & $x$ & $x$ & $x$ & $x$ & & $x$ & & $x$ & & $x$ & $x$ & $x$ & & $x$ & $x$ & $x$ & $x$ & & $x$ & $x ;$ & $x$ & & $x$ & $x$ & & $x x$ & $x \times$ & & $x$ & $\Sigma$ & $x>$ & $x$ \\
\hline$\frac{2}{4}$ & & $x$ & $x$ & $x$ & & $x$ & & $x$ & $x$ & $x$ & & $x$ & $x$ & $x$ & & $x$ & $x$ & $x$ & $x$ & $x$ & $x$ & $x ;$ & $x$ & & $x$ & $x$ & $x$ & $x x$ & $x \times$ & & $x$ & $x$ & $x>$ & $x$ \\
\hline を & & $x$ & $x$ & & & & & & & & & & $x$ & $x$ & & $x$ & $x$ & $x$ & $x$ & & $x$ & & $x$ & & $x$ & & $x$ & $x$ & $x \times$ & & $x$ & $x$ & & \\
\hline 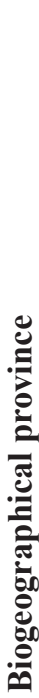 & 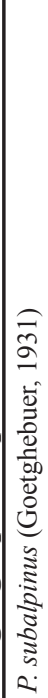 & 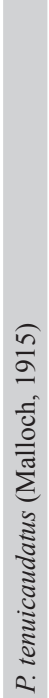 & 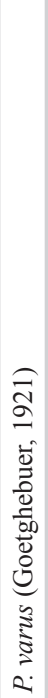 & 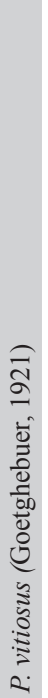 & 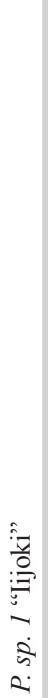 & 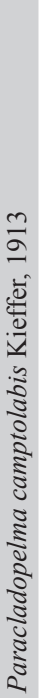 & 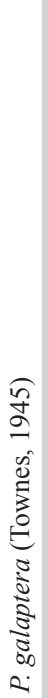 & 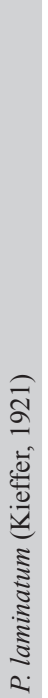 & 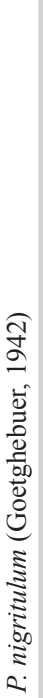 & 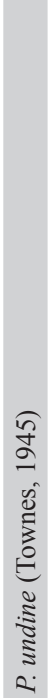 & 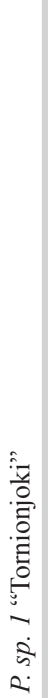 & 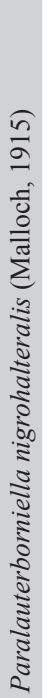 & 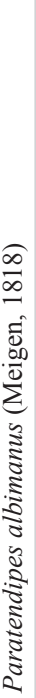 & 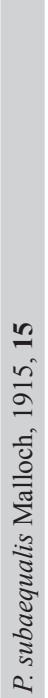 & 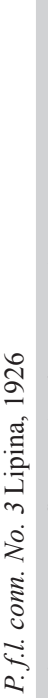 & 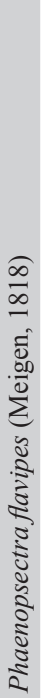 & 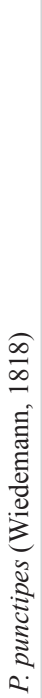 & 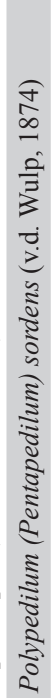 & 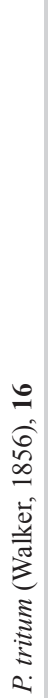 & 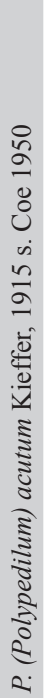 & 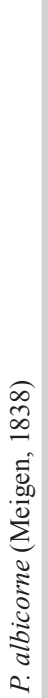 & 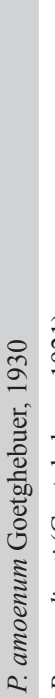 & 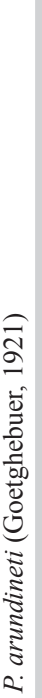 & 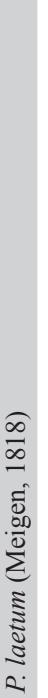 & 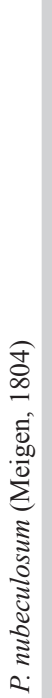 & 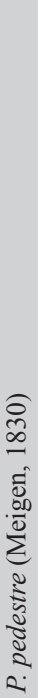 & 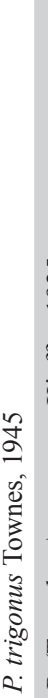 & 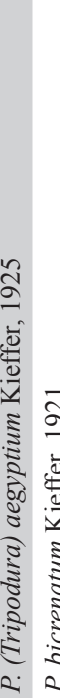 & 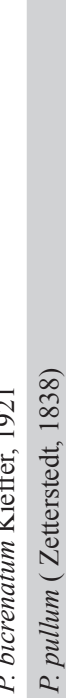 & 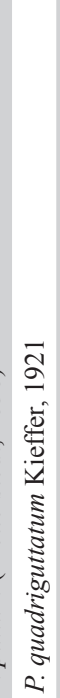 & 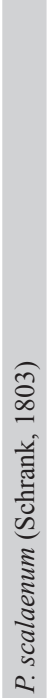 & 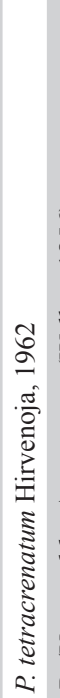 & 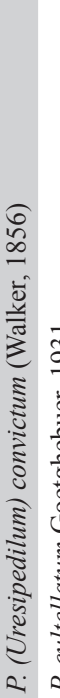 & 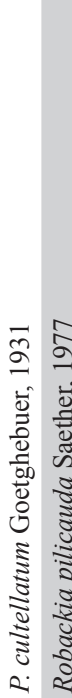 \\
\hline
\end{tabular}




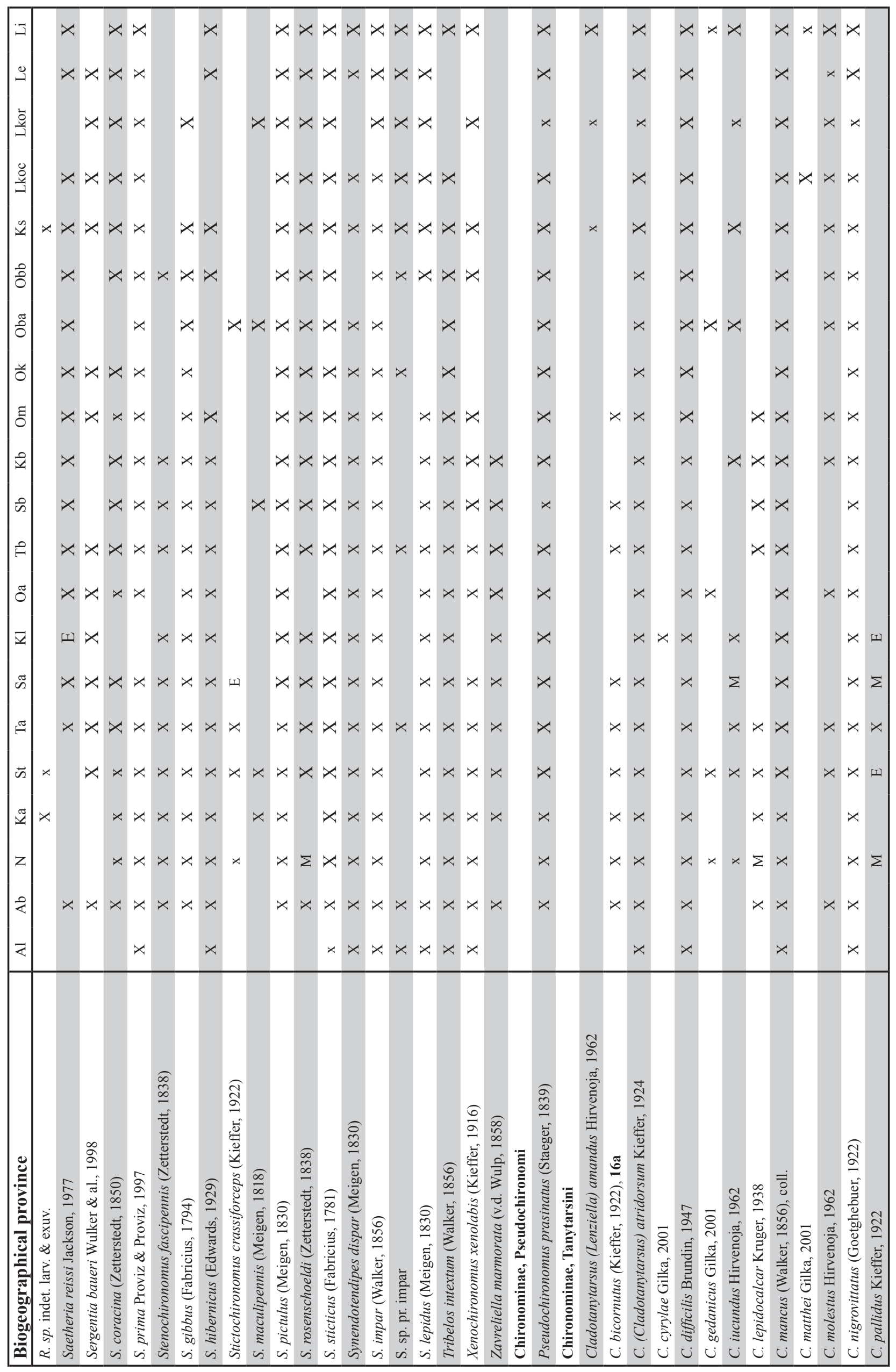




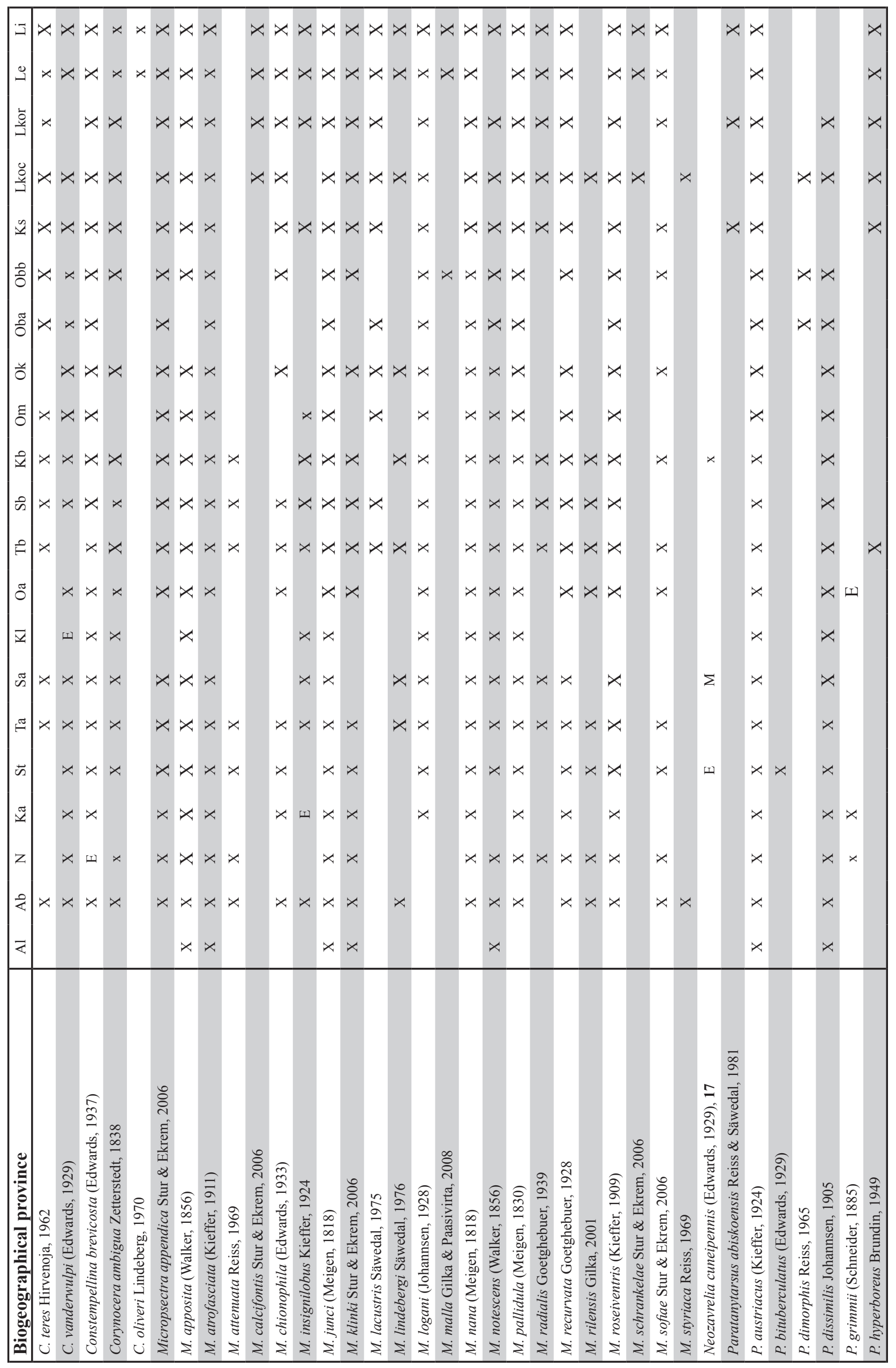




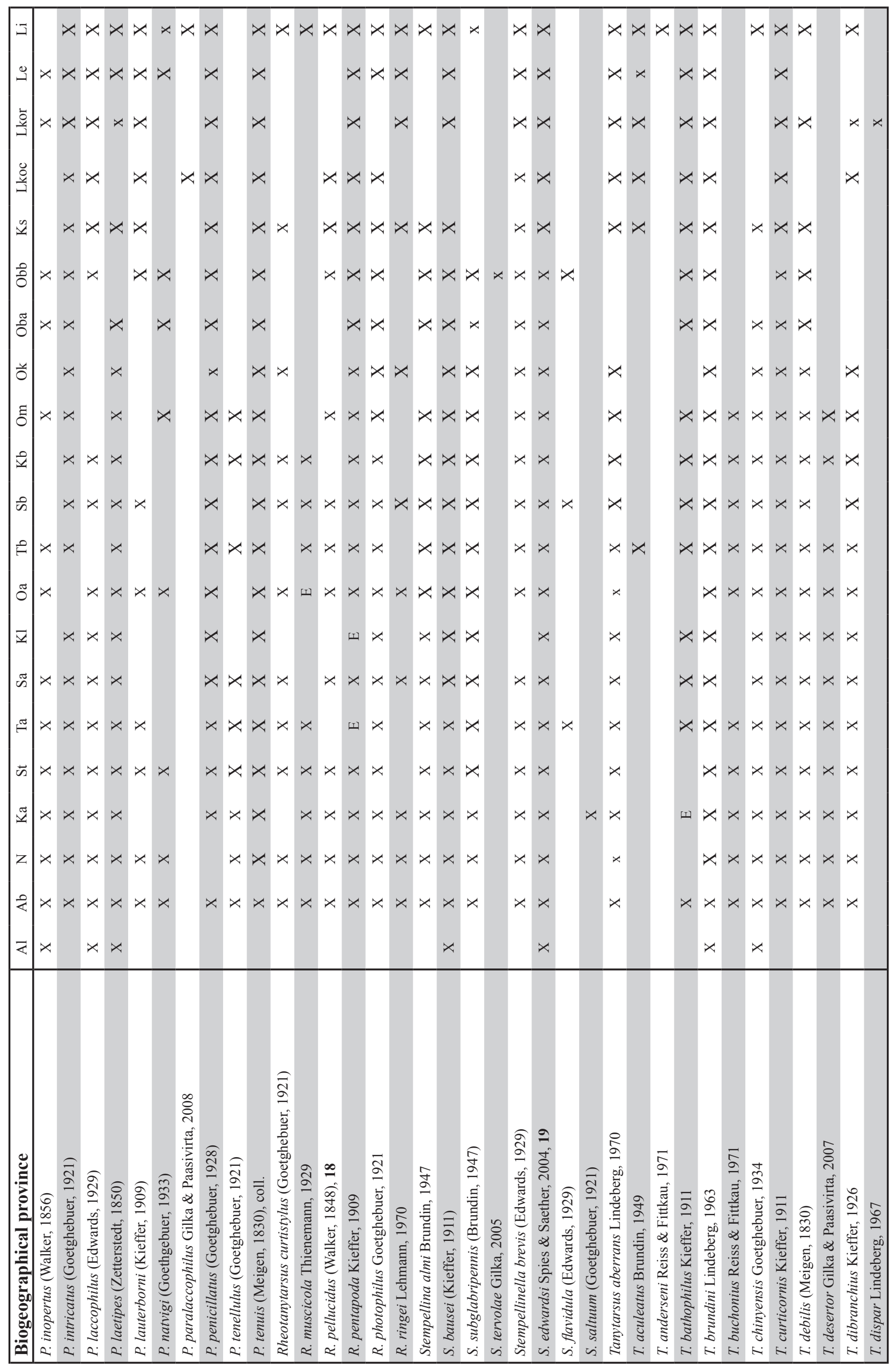




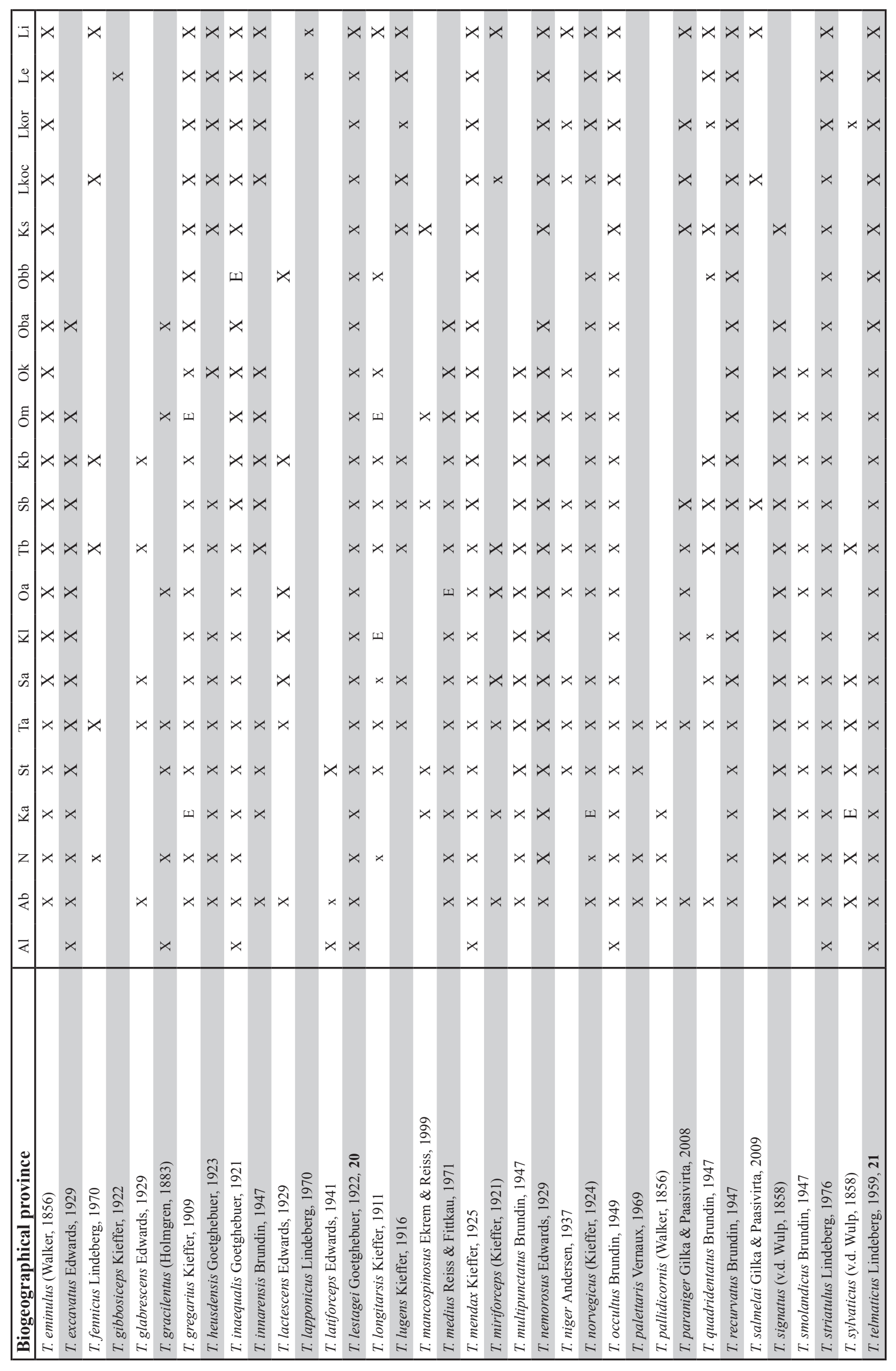




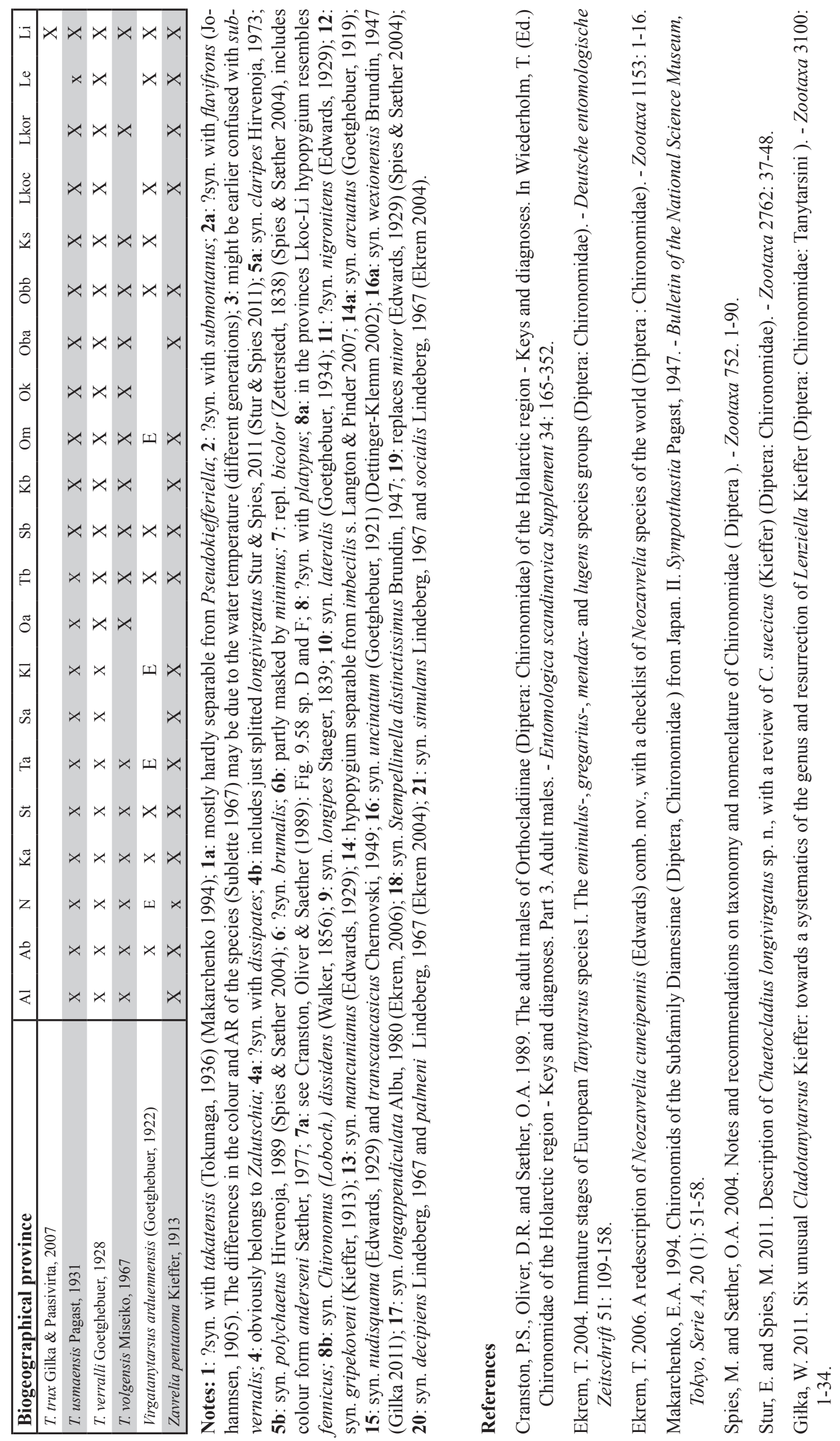

